\title{
From the simple reacting sphere kinetic model to the reaction-diffusion system of Maxwell-Stefan type
}

\author{
Benjamin Anwasia $^{a) *}$, Patrícia Gonçalves ${ }^{b) \dagger}$, Ana Jacinta Soares ${ }^{a) \ddagger}$ \\ ${ }^{a)}$ Centro de Matemática, Universidade do Minho, Braga, Portugal \\ ${ }^{b)}$ Center for Mathematical Analysis, Geometry and Dynamical Systems, \\ Instituto Superior Técnico, Universidade de Lisboa, Portugal
}

(accepted in Dec 2018 for publication in Comm. Math. Sci.)

\begin{abstract}
In this paper we perform a formal asymptotic analysis on a kinetic model for reactive mixtures in order to derive a reaction-diffusion system of Maxwell-Stefan type. More specifically, we start from the kinetic model of simple reacting spheres for a quaternary mixture of monatomic ideal gases that undergoes a reversible chemical reaction of bimolecular type. Then, we consider a scaling describing a physical situation in which mechanical collisions play a dominant role in the evolution process, while chemical reactions are slow, and compute explicitly the production terms associated to the concentration and momentum balance equations for each species in the reactive mixture. Finally, we prove that, under the isothermal assumption, the limit equations for the scaled kinetic model is the reaction diffusion system of Maxwell-Stefan type.
\end{abstract}

Keywords: Boltzmann-type equations. Chemically reactive mixtures. Diffusion limit. Kinetic theory of gases. Maxwell-Stefan equations.

AMS Subject Classification: 82C40, 76P05, 80A32, 35Q20.

\section{Introduction}

The description and modelling of chemically reactive mixtures is a topic of great importance due to many engineering applications related, for example, to chemical industry and biotechnology [17, 35, 39]. In particular, a proper description of diffusive phenomena in multicomponent mixtures, with or without chemical reactions, is crucial in many simulations and design processes used by chemical engineers [27. In this context, the Maxwell-Stefan (MS) equations are used by many applied and experimental researchers to model and predict diffusion as well as mass transfer processes in multicomponent mixtures [35, 36, 39. In fact, it is well known that the MS equations are adequate to describe non-typical diffusions that appear as a consequence of some thermodynamic non-idealities, by introducing the chemical potential gradients as driving forces [27, 39]. When a multicomponent mixture with chemical reaction is considered, a hydrodynamic system which consists of the continuity equations for the constituents in the mixture and the MS equations, can be used to describe diffusion among the constituents and how their concentrations change as a consequence of the chemical reaction.

*id62260alunos . uminho.pt

patricia.goncalves@math.tecnico.ulisboa.pt

tajsoares@math.uminho.pt 
Despite the practical interest and applications of the MS equations for multicomponent mixtures with or without chemical reaction, not much is known about the mathematical analysis of these equations. Rigorous results have been published over the past few years, see [8, 11, 12, 13, 21, 22, 23. In particular, 12, 13, 22 dealt with the formal derivation of hydrodynamic systems of MS equations coupled with the continuity equations for the species from a kinetic (mesoscopic) system of Boltzmann equations for non-reactive multicomponent mixture and obtained explicit expressions for the diffusion coefficients in terms of the kinetic model parameters. Such papers follow the well established line of research initiated by Golse and co-workers [2, 3, 4] on the transition from kinetic Boltzmann models to hydrodynamic equations of fluid mechanics. In close connection with these works, but considering chemically reactive mixtures, we quote here [5, 6, 7] which dealt with the derivation of macroscopic reaction-diffusion equations from a system of reactive Boltzmann equations. In these papers, using an appropriate scaling of the reactive Boltzmann equations and assuming different types of molecular interactions, the evolution equations for the species number densities were explicitly derived in the asymptotic limit of small Knudsen number. Moreover, the convergence from the reactive Boltzmann equations to the reaction-diffusion system was proven and discussed in [5, 6, Reaction-diffusion equations for chemically reactive mixtures were also derived in 33, 34, 40, starting from kinetic equations of Fokker-Planck type. Various scalings were considered in view of analyzing the interactions between transport processes and chemical reactions.

In the present paper, we are interested in the limiting process that leads from a particular model of reactive Boltzmann equations to a reaction-diffusion system of MS type. More precisely, we consider a quaternary mixture of monatomic ideal gases undergoing a bimolecular reversible reaction, described by the simple reacting sphere (SRS) kinetic model [30, 32, 38, in which both elastic and reactive collisions are of hard-sphere type. Then, considering a scaling of the SRS kinetic equations for which elastic collisions are dominant and reactive collisions are less frequent, and assuming isothermal condition, we formally derive the reaction-diffusion system of MS type form the evolution of the number density and momentum balance of the species. The formal derivation of these equations from a kinetic model is our main contribution in this paper and, as far as we know, this is the first attempt in this direction. Our assumptions correspond to a regime of slow, isothermal chemical reaction that occurs when the chemical process takes place at a rate slow enough to allow the surroundings to continuously compensate for the balance of reaction heat between the reactants and products 8, 9, 10, 21, 43, 44. In the limit, we obtain a reaction diffusion system similar to those studied in [8, 9, 10. If the isothermal assumption is disregarded, the evolution equation for the temperature of the mixture should be considered and the reaction diffusion system obtained in the limit will be similar to the one obtained in [42] for a non-reactive mixture.

After this introduction, the remaining part of our work is organized as follows. The reaction-diffusion system of MS type for a multi-species reactive mixture in the context of continuum mechanics is introduced in Section 2. In Section 3 we describe the SRS kinetic model and introduce its relevant properties that are essential for our analysis.

The diffusive asymptotics of the SRS kinetic model towards the reaction-diffusion system of MS type is studied in Section 4, after a proper scaling of the SRS equations. Our conclusions and some future perspectives are stated in Section 5 . Finally, we include an Appendix in Section 6 where we give some steps and other details about the computation of the integrals appearing in Section 4

\section{The continuum reaction-diffusion system of Maxwell-Stefan type}

In this section, we introduce a mathematical model for a reactive multi-species gaseous mixture in the context of continuum mechanics. The mixture is influenced by two processes, namely diffusion, which causes the species to spread in space, and chemical reaction, which results in the transformation of the species into each other. The model equations consist of the concentration balance equations for the reactive species in the mixture coupled with the MS equations for the momentum of the species. These equations describe how both processes affect the evolution of the mixture and will be referred to as the reaction-diffusion system of MS type.

Let $\Omega \subset \mathbb{R}^{3}$ be a bounded domain with boundary denoted by $\partial \Omega$ and outward normal vector at each point $\mathbf{x}$ of the boundary given by $\nu(\mathbf{x})$. We consider a mixture of four species, say $A_{1}, A_{2}, A_{3}$ and $A_{4}$, that participate in a chemical reaction of type

$$
A_{1}+A_{2} \rightleftharpoons A_{3}+A_{4} .
$$


This means that species $A_{1}, A_{2}$ react to produce species $A_{3}, A_{4}$ and conversely, species $A_{3}, A_{4}$ also react to produce species $A_{1}, A_{2}$. We say that $A_{1}, A_{2}$ and $A_{3}, A_{4}$ are the reactive species (or the reactive pairs), more specifically $A_{1}, A_{2}$ are reactants and $A_{3}, A_{4}$ are products of the forward chemical reaction. For each species $A_{i}$, with $i=1,2,3,4$, let $\varrho_{i}(t, \mathbf{x}) \geq 0$ be the mass density, $\mathbf{u}_{i}(t, \mathbf{x})$ the mean velocity and $r_{i}(t, \mathbf{x})$ the production rate of mass density due to the chemical reaction, where $\mathbf{x} \in \Omega$ and $t>0$.

The mass balance equation for each constituent in the reactive mixture reads

$$
\frac{\partial \varrho_{i}}{\partial t}+\frac{\partial}{\partial \mathbf{x}}\left(\varrho_{i} \mathbf{u}_{i}\right)=r_{i}, \quad \mathbf{x} \in \Omega, \quad t>0
$$

Due to the type of chemical reaction (1), the production rates satisfy the condition

$$
\sum_{i=1}^{4} r_{i}=0
$$

which results in the conservation of the total mass of the mixture.

In this paper, we adopt a molar based description (see [16]) of the reactive mixture and, as a result, for each species $A_{i}$ we introduce the molar mass $M_{i}$ and define the molar concentration $c_{i}(t, \mathbf{x})$ and its production rate $\mathcal{J}_{i}$, the molar flux $\mathbf{N}_{i}(t, \mathbf{x})$, the mole fraction $\gamma_{i}(t, \mathbf{x})$ and the molar diffusive flux $\mathbf{J}_{i}(t, \mathbf{x})$, given respectively by

$$
c_{i}=\frac{\varrho_{i}}{M_{i}}, \quad \mathcal{J}_{i}=\frac{r_{i}}{M_{i}}, \quad \mathbf{N}_{i}=c_{i} \mathbf{u}_{i}, \quad \gamma_{i}=\frac{c_{i}}{c}, \quad \mathbf{J}_{i}=c_{i}\left(\mathbf{u}_{i}-\mathbf{v}\right) .
$$

Here, $c:=c(t, \mathbf{x})$ and $\mathbf{v}:=\mathbf{v}(t, \mathbf{x})$ are the molar concentration and molar average velocity of the mixture, defined by

$$
c=\sum_{i=1}^{4} c_{i}, \quad \mathbf{v}=\sum_{i=1}^{4} \gamma_{i} \mathbf{u}_{i}, \quad \mathbf{x} \in \Omega, \quad t>0 .
$$

Note that the molar diffusive fluxes $\mathbf{J}_{i}$ satisfy the constraint

$$
\sum_{i=1}^{4} \mathbf{J}_{i}=0, \quad \mathbf{x} \in \Omega, \quad t>0 .
$$

In our analysis, diffusion and chemical reaction are the relevant effects in the mixture. Therefore, as in 8, 9, 10, 21, we neglect the effects due to temperature gradients, by assuming an isothermal condition which corresponds to a uniform in space and constant in time mixture temperature $T$. We also neglect the effects due to convection and advection.

From the mass balance equation (2), using definitions (4), we obtain the evolution equations for the species concentrations $c_{i}$ in the form

$$
\frac{\partial c_{i}}{\partial t}+\frac{\partial \mathbf{J}_{i}}{\partial \mathbf{x}}=\mathcal{J}_{i}, \quad \mathbf{x} \in \Omega, \quad t>0, \quad i=1,2,3,4,
$$

where we have considered $\frac{\partial}{\partial \mathbf{x}}\left(c_{i} \mathbf{v}\right)=\mathbf{0}$, since convection is neglected.

The diffusion process in multicomponent gaseous mixtures can be accurately described by the MS equations, which express the relationship between the molar diffusive fluxes and the chemical potentials of the species. Adopting the standard form for the chemical potentials [21] and taking into account the isothermal assumption, the driving forces (given by the chemical potentials) become equal to the concentration gradients of the species and the MS equations relate the molar diffusive fluxes to the concentration gradients. Following the description of [35, and using our notation, the MS equations under isobaric assumption (i.e. constant pressure) can be written in the form

$$
\frac{\partial c_{i}}{\partial \mathbf{x}}=-\frac{1}{c} \sum_{\substack{s=1 \\ s \neq i}}^{4} \frac{c_{s} \mathbf{J}_{i}-c_{i} \mathbf{J}_{s}}{D_{i s}}, \quad \mathbf{x} \in \Omega, \quad t>0, \quad i=1,2,3,4,
$$


where $D_{i s}$ is the diffusion coefficient associated to species $A_{i}$ and $A_{s}$, with $D_{i s}=D_{s i}$. Observe that summing (7) over all species as well as (8) over all species, we obtain

$$
\frac{\partial c}{\partial t}=0 \quad \text { and } \quad \frac{\partial c}{\partial \mathbf{x}}=0,
$$

which means that the total molar concentration of the mixture, $c$, is uniform in $\mathbf{x}$ and constant in $t$. Also, the MS equations (8) are linearly dependent and only three of these equations are independent, so we have to add another equation to the system.

For what concerns the boundary conditions to join to our set of equations, we assume that the chemical reaction (1) takes place in a closed domain, so that we impose

$$
\nu \cdot \mathbf{J}_{i}=0, \quad \mathbf{x} \in \partial \Omega, \quad t>0, \quad i=1,2,3,4 .
$$

Equations (7) and (8), together with constraint (6), constitute the reaction-diffusion system of MS type. These equations are similar to those studied in $[8,23$. They are used to describe diffusion and chemical kinetics of the multi-species reactive mixture in the context of continuum mechanics.

The aim of the present paper is to formally derive the balance equations (7) and the MS equations (8) as the hydrodynamic limit of the SRS kinetic model for the considered reactive mixture. The chemical production rates $\mathcal{J}_{i}$ and the diffusion coefficients $D_{i s}$ will be explicitly computed from the collisional dynamics of the kinetic model and will be expressed in terms of some kinetic parameters.

\section{The SRS kinetic model}

In this section, we introduce our kinetic model for the quaternary reactive mixture considered in Section 2, This model is based on the kinetic theory of simple reacting spheres (SRS), first proposed by Marron in [28, and then developed by Xystris, Dahler and Qin in [15, 32, 38. Some aspects of the mathematical analysis of the SRS model were investigated, for example, in [14, 20, 29, 30. Here, we introduce the model and briefly describe some of its properties needed for the analysis developed in this paper. Other details about the SRS model can be seen in the references just quoted above.

We consider the quaternary reactive mixture introduced in Section 2 , whose constituents $A_{1}, A_{2}, A_{3}, A_{4}$ participate in the bimolecular chemical reaction (1) and confined ourselves to the simplifying assumption that the species are endowed with only translational degree of freedom. In other words, the species are monatomic like (i.e. internal degrees of freedom associated to rotational, vibrational and nuclei energies are not taken into account. For each $i=1,2,3,4$, let $m_{i}, d_{i}$ and $E_{i}$ be, respectively, the mass, the diameter and the formation energy of the species $A_{i}$. Conservation of mass holds for the chemical reaction (1) and thus we have

$$
m_{1}+m_{2}=m_{3}+m_{4}=M
$$

The reaction heat, denoted by $Q_{R}$, is given by the difference between the formation energies of the products of the forward reaction and those of the reactants, i.e.

$$
Q_{R}=E_{3}+E_{4}-E_{1}-E_{2} .
$$

This means that the forward reaction $A_{1}+A_{2} \rightarrow A_{3}+A_{4}$ is exothermic if $Q_{R}<0$. Otherwise, it is endothermic. Also, we introduce the activation energy $\zeta_{i}$ for each of the species $A_{i}$, such that $\zeta_{1}=\zeta_{2}, \zeta_{3}=\zeta_{4}$ and $\zeta_{3}=\zeta_{1}-Q_{R}$.

\subsection{Collisional dynamics}

Particles in the mixture undergo binary elastic collisions and reactive encounters obeying the chemical law (1), both of hard sphere type. Elastic collisions take place between particles of the same species, as well as between 
particles of different species. The cross section of an elastic collision between particles of species $A_{i}, A_{s}$ is defined by

$$
\sigma_{i s}^{2}=\frac{1}{4}\left(d_{i}+d_{s}\right)^{2}, \quad i, s=1,2,3,4 \text {. }
$$

If $\mathbf{v}_{i}, \mathbf{v}_{s}$ are the pre-collisional velocities and $\mathbf{v}_{i}^{\prime}, \mathbf{v}_{s}^{\prime}$ the post-collisional velocities, the conservation laws of linear momentum and kinetic energy for elastic collisions are, respectively, given by

$$
m_{i} \mathbf{v}_{i}+m_{s} \mathbf{v}_{s}=m_{i} \mathbf{v}_{i}^{\prime}+m_{s} \mathbf{v}_{s}^{\prime} \quad \text { and } \quad m_{i}\left(\mathbf{v}_{i}\right)^{2}+m_{s}\left(\mathbf{v}_{s}\right)^{2}=m_{i}\left(\mathbf{v}_{i}^{\prime}\right)^{2}+m_{s}\left(\mathbf{v}_{s}^{\prime}\right)^{2}
$$

The post-collisional velocities are given in terms of the pre-collisional velocities by

$$
\mathbf{v}_{i}^{\prime}=\mathbf{v}_{i}-2 \frac{\mu_{i s}}{m_{i}} \epsilon\left\langle\epsilon, \mathbf{v}_{i}-\mathbf{v}_{s}\right\rangle \quad \text { and } \quad \mathbf{v}_{s}^{\prime}=\mathbf{v}_{s}+2 \frac{\mu_{i s}}{m_{s}} \epsilon\left\langle\epsilon, \mathbf{v}_{i}-\mathbf{v}_{s}\right\rangle
$$

where

$$
\mu_{i s}=\frac{m_{i} m_{s}}{m_{i}+m_{s}}
$$

is the reduced mass of the colliding pair, $\epsilon$ is a unit vector directed along the line joining the centre of the two spheres at the moment of impact, that is $\epsilon \in \mathbb{S}_{+}^{2}=\left\{\bar{\epsilon} \in \mathbb{R}^{3}:\|\bar{\epsilon}\|=1,\left\langle\bar{\epsilon}, \mathbf{v}_{i}-\mathbf{v}_{s}\right\rangle>0\right\}$. Moreover, $\langle\cdot, \cdot\rangle$ represents the inner product in $\mathbb{R}^{3}$ and $\|\cdot\|$ is the norm induced by this inner product. For convenience, we introduce the total mass of the colliding pair, $M_{i s}=m_{i}+m_{s}$.

Remark 3.1 Note that if we use spherical coordinates with $\theta \in[0, \pi / 2]$ as the polar angle between $\mathbf{v}_{i}-\mathbf{v}_{s}$ and $\epsilon$ and $\phi \in\left[0,2 \pi\left[\right.\right.$ as the azimuthal angle in the plane orthogonal to $\mathbf{v}_{i}-\mathbf{v}_{s}$, then $\epsilon=(\sin \theta \cos \phi, \sin \theta \sin \phi, \cos \theta)$. Also, $\left\langle\epsilon, \mathbf{v}_{i}-\mathbf{v}_{s}\right\rangle=V^{*} \cos \theta$, where $V^{*}=\left\|\mathbf{v}_{i}-\mathbf{v}_{s}\right\|$ is the norm of the relative velocity before collision.

Concerning reactive encounters, a collision between particles of species $A_{i}, A_{j}$ with pre-collisional velocities $\mathbf{v}_{i}, \mathbf{v}_{j}$ will result in a chemical reaction if the kinetic energy associated with the relative motion of the colliding pair along the line of their centres is greater than, or equal to, the activation energy, that is

$$
\frac{1}{2} \mu_{i j}\left\langle\epsilon, \mathbf{v}_{i}-\mathbf{v}_{j}\right\rangle^{2} \geq \zeta_{i}
$$

If $A_{k}, A_{l}$ represent the products of the forward reaction and $\mathbf{v}_{k}^{\circ}, \mathbf{v}_{l}^{\circ}$ their post-collisional velocities, then the conservation laws of linear momentum and total energy (kinetic plus binding) for reactive collisions are, respectively, given by

$$
\begin{aligned}
m_{i} \mathbf{v}_{i}+m_{j} \mathbf{v}_{j} & =m_{k} \mathbf{v}_{k}^{\circ}+m_{l} \mathbf{v}_{l}^{\circ} \\
E_{i}+\frac{1}{2} m_{i}\left(\mathbf{v}_{i}\right)^{2}+E_{j}+\frac{1}{2} m_{j}\left(\mathbf{v}_{j}\right)^{2} & =E_{k}+\frac{1}{2} m_{k}\left(\mathbf{v}_{k}^{\circ}\right)^{2}+E_{l}+\frac{1}{2} m_{l}\left(\mathbf{v}_{l}^{\circ}\right)^{2}
\end{aligned}
$$

where the indexes are such that $(i, j, k, l) \in\{(1,2,3,4),(2,1,4,3),(3,4,1,2),(4,3,2,1)\}$. From now on, if nothing is said about the indexes $(i, j, k, l)$, we assume that they are as introduced above.

Remark 3.2 From condition (16), for a reactive collision to occur, we must have $\left\langle\epsilon, \mathbf{v}_{i}-\mathbf{v}_{j}\right\rangle \geq \sqrt{2 \zeta_{i} / \mu_{i j}}$. Using the definitions of the unit vector $\epsilon$ and relative velocity norm $V=\left\|\mathbf{v}_{i}-\mathbf{v}_{j}\right\|$ before the collision, we obtain $V \geq \sqrt{2 \zeta_{i} / \mu_{i j}}$, which motivates the definition of the threshold relative velocity as $\Xi_{i j}=\sqrt{2 \zeta_{i} / \mu_{i j}}$. In particular, $\Xi_{i j}$ is the required relative velocity necessary to assure that the collision will be of reactive type.

The reactive cross sections for the direct and reverse chemical reactions can be defined in terms of their threshold relative velocities by

$$
\sigma_{12}^{\prime 2}=\left\{\begin{array}{ll}
\beta_{12} \sigma_{12}^{2}, & \left\langle\epsilon, \mathbf{v}_{1}-\mathbf{v}_{2}\right\rangle \geq \Xi_{12}, \\
0, & \left\langle\epsilon, \mathbf{v}_{1}-\mathbf{v}_{2}\right\rangle<\Xi_{12},
\end{array} \quad \sigma_{34}^{\prime 2}= \begin{cases}\beta_{34} \sigma_{34}^{2}, & \left\langle\epsilon, \mathbf{v}_{3}-\mathbf{v}_{4}\right\rangle \geq \Xi_{34}, \\
0, & \left\langle\epsilon, \mathbf{v}_{3}-\mathbf{v}_{4}\right\rangle<\Xi_{34},\end{cases}\right.
$$


where the coefficients $\beta_{i j}$ represent the fraction of colliding pairs with enough kinetic energy to produce a reaction that in fact react chemically. They play the role of steric factors, with $0 \leq \beta_{i j} \leq 1$.

The post-collisional velocities for the forward chemical reaction $A_{1}+A_{2} \rightarrow A_{3}+A_{4}$ are given by

$$
\begin{aligned}
& \mathbf{v}_{3}^{\circ}=\frac{1}{M}\left[m_{1} \mathbf{v}_{1}+m_{2} \mathbf{v}_{2}+m_{4} \sqrt{\frac{\mu_{12}}{\mu_{34}}}\left\{\left(\mathbf{v}_{1}-\mathbf{v}_{2}\right)-\epsilon\left\langle\epsilon,\left(\mathbf{v}_{1}-\mathbf{v}_{2}\right)\right\rangle+\epsilon \omega^{-}\right\}\right], \\
& \mathbf{v}_{4}^{\circ}=\frac{1}{M}\left[m_{1} \mathbf{v}_{1}+m_{2} \mathbf{v}_{2}-m_{3} \sqrt{\frac{\mu_{12}}{\mu_{34}}}\left\{\left(\mathbf{v}_{1}-\mathbf{v}_{2}\right)-\epsilon\left\langle\epsilon,\left(\mathbf{v}_{1}-\mathbf{v}_{2}\right)\right\rangle+\epsilon \omega^{-}\right\}\right],
\end{aligned}
$$

where $\omega^{-}=\sqrt{\left(\left\langle\epsilon,\left(\mathbf{v}_{1}-\mathbf{v}_{2}\right)\right\rangle\right)^{2}-2 Q_{R} / \mu_{12}}$. Analogously, the post-collisional velocities for the reverse chemical reaction $A_{3}+A_{4} \rightarrow A_{1}+A_{2}$ are given by

$$
\begin{aligned}
& \mathbf{v}_{1}^{\circ}=\frac{1}{M}\left[m_{3} \mathbf{v}_{3}+m_{4} \mathbf{v}_{4}+m_{2} \sqrt{\frac{\mu_{34}}{\mu_{12}}}\left\{\left(\mathbf{v}_{3}-\mathbf{v}_{4}\right)-\epsilon\left\langle\epsilon,\left(\mathbf{v}_{3}-\mathbf{v}_{4}\right)\right\rangle+\epsilon \omega^{+}\right\}\right], \\
& \mathbf{v}_{2}^{\circ}=\frac{1}{M}\left[m_{3} \mathbf{v}_{3}+m_{4} \mathbf{v}_{4}-m_{1} \sqrt{\frac{\mu_{34}}{\mu_{12}}}\left\{\left(\mathbf{v}_{3}-\mathbf{v}_{4}\right)-\epsilon\left\langle\epsilon,\left(\mathbf{v}_{3}-\mathbf{v}_{4}\right)\right\rangle+\epsilon \omega^{+}\right\}\right],
\end{aligned}
$$

where $\omega^{+}=\sqrt{\left(\left\langle\epsilon,\left(\mathbf{v}_{3}-\mathbf{v}_{4}\right)\right\rangle\right)^{2}+2 Q_{R} / \mu_{34}}$.

We close this subsection by recalling some properties about the dynamics of the reactive collisions that have been established in 14 and [30.

Property 3.3 For a reactive collision, the following properties hold

$$
\begin{aligned}
& \frac{1}{2} \mu_{i j}\left(\mathbf{v}_{i}-\mathbf{v}_{j}\right)^{2}=\frac{1}{2} \mu_{k l}\left(\mathbf{v}_{k}^{\circ}-\mathbf{v}_{l}^{\circ}\right)^{2}+Q_{R}, \\
& \mu_{i j}\left(\left\langle\epsilon, \mathbf{v}_{i}-\mathbf{v}_{j}\right\rangle\right)^{2}=\mu_{k l}\left(\left\langle\epsilon, \mathbf{v}_{k}^{\circ}-\mathbf{v}_{l}^{\circ}\right\rangle\right)^{2}+2 Q_{R}, \\
& \frac{1}{2} \mu_{i j}\left(\left\langle\epsilon, \mathbf{v}_{i}-\mathbf{v}_{j}\right\rangle\right)^{2}-\zeta_{i}=\frac{1}{2} \mu_{k l}\left(\left\langle\epsilon, \mathbf{v}_{k}^{\circ}-\mathbf{v}_{l}^{\circ}\right\rangle\right)^{2}-\zeta_{k}, \\
& \left\langle\epsilon, \mathbf{v}_{i}-\mathbf{v}_{j}\right\rangle=\left(\frac{\mu_{k l}}{\mu_{i j}}\right)^{\frac{1}{2}} \omega^{+} .
\end{aligned}
$$

Property 3.4 For a fixed vector $\epsilon$, the Jacobians of the transformations $\left(\mathbf{v}_{i}, \mathbf{v}_{j}\right) \mapsto\left(\mathbf{v}_{k}^{\circ}, \mathbf{v}_{l}^{\circ}\right)$ and $\left(\mathbf{v}_{k}, \mathbf{v}_{l}\right) \mapsto$ $\left(\mathbf{v}_{i}^{\circ}, \mathbf{v}_{j}^{\circ}\right)$ are, respectively, given by

$$
\left(\frac{\mu_{k l}}{\mu_{i j}}\right)^{\frac{3}{2}} \frac{\left\langle\epsilon, \mathbf{v}_{k}^{\circ}-\mathbf{v}_{l}^{\circ}\right\rangle}{\omega^{+}} \text {and }\left(\frac{\mu_{i j}}{\mu_{k l}}\right)^{\frac{3}{2}} \frac{\left\langle\epsilon, \mathbf{v}_{i}-\mathbf{v}_{j}\right\rangle}{\omega^{-}} .
$$

\section{$3.2 \quad$ Kinetic equations}

The state of the reactive mixture is described by the one-particle distribution functions $f_{i}\left(t, \mathbf{x}, \mathbf{v}_{i}\right)$ representing the density of particles of species $A_{i}$, expressed in moles, which at time $t$ are located at position $\mathbf{x}$ and have velocity $\mathbf{v}_{i}$, with $i=1,2,3,4$ and $\left(t, \mathbf{x}, \mathbf{v}_{i}\right) \in \mathbb{R}_{+} \times \Omega \times \mathbb{R}^{3}$. The functions $f_{i}$ are related to the molar concentrations $c_{i}$ through the following expressions

$$
c_{i}(t, \mathbf{x})=\int_{\mathbb{R}^{3}} f_{i}\left(t, \mathbf{x}, \mathbf{v}_{i}\right) d \mathbf{v}_{i}, \quad t \geq 0, \mathbf{x} \in \Omega, \quad i=1,2,3,4 .
$$

In absence of external forces, the SRS kinetic equations are given by

$$
\frac{\partial f_{i}}{\partial t}+\mathbf{v}_{i} \cdot \frac{\partial f_{i}}{\partial \mathbf{x}}=J_{i}, \quad \text { in } \quad \mathbb{R}_{+} \times \Omega \times \mathbb{R}^{3},
$$


for $i=1,2,3,4$, with $J_{i}=J_{i}^{E}+J_{i}^{R}$, where $J_{i}^{E}$ is the elastic collision operator and $J_{i}^{R}$ is the reactive collision operator. They are respectively defined as follows,

$$
\begin{aligned}
J_{i}^{E}=\sigma_{i i}^{2} \int_{\mathbb{R}^{3}} \int_{\mathbb{S}_{+}^{2}}\left[f_{i}{ }^{\prime} f_{i_{*}}^{\prime}-f_{i} f_{i_{*}}\right]\left\langle\epsilon, \mathbf{v}_{i}-\mathbf{v}_{i_{*}}\right\rangle d \epsilon d \mathbf{v}_{i_{*}} \\
+\sum_{\substack{s=1 \\
s \neq i}}^{4} \sigma_{i s}^{2} \int_{\mathbb{R}^{3}} \int_{\mathbb{S}_{+}^{2}}\left[f_{i}{ }^{\prime} f_{s}^{\prime}-f_{i} f_{s}\right]\left\langle\epsilon, \mathbf{v}_{i}-\mathbf{v}_{s}\right\rangle d \epsilon d \mathbf{v}_{s} \\
\quad-\beta_{i j} \sigma_{i j}^{2} \int_{\mathbb{R}^{3}} \int_{\mathbb{S}_{+}^{2}}\left[f_{i}{ }^{\prime} f_{j}{ }^{\prime}-f_{i} f_{j}\right] \Theta\left(\left\langle\epsilon, \mathbf{v}_{i}-\mathbf{v}_{j}\right\rangle-\Xi_{i j}\right)\left\langle\epsilon, \mathbf{v}_{i}-\mathbf{v}_{j}\right\rangle d \epsilon d \mathbf{v}_{j}, \\
J_{i}^{R}=\beta_{i j} \sigma_{i j}^{2} \int_{\mathbb{R}^{3}} \int_{\mathbb{S}_{+}^{2}}\left[\left(\frac{\mu_{i j}}{\mu_{k l}}\right)^{2} f_{k}^{\circ} f_{l}^{\circ}-f_{i} f_{j}\right] \Theta\left(\left\langle\epsilon, \mathbf{v}_{i}-\mathbf{v}_{j}\right\rangle-\Xi_{i j}\right)\left\langle\epsilon, \mathbf{v}_{i}-\mathbf{v}_{j}\right\rangle d \epsilon d \mathbf{v}_{j},
\end{aligned}
$$

where we have adopted the usual notation $f_{i}^{\prime}=f\left(t, \mathbf{x}, \mathbf{v}_{i}^{\prime}\right), f_{i *}^{\prime}=f\left(t, \mathbf{x}, \mathbf{v}_{i *}^{\prime}\right), f_{s}^{\prime}=f\left(t, \mathbf{x}, \mathbf{v}_{s}^{\prime}\right), f_{k}^{\circ}=f\left(t, \mathbf{x}, \mathbf{v}_{k}^{\circ}\right)$, $f_{l}^{\circ}=f\left(t, \mathbf{x}, \mathbf{v}_{l}^{\circ}\right)$, and $\Theta$ is a Heaviside step function, defined at $x \in \mathbb{R}$ by

$$
\Theta(x)= \begin{cases}1, & x \geq 0 \\ 0, & x<0\end{cases}
$$

Equations (28) together with expressions (29) and (30) constitute the SRS kinetic system. Without being precise, the accompanying boundary conditions to describe the interactions between the molecules and the boundary $\partial \Omega$ of the evolution domain are taken to be of specular reflection type [37. Such boundary conditions ensure that the reactive mixture is considered in a closed domain, as assumed in Section 2

In expression 290 for the elastic collision operator, the first term on the right hand side represents collisions involving particles of the same species and the index $i_{*}$ is used to distinguish their velocities. This term represents the standard Boltzmann collision operator for a single gas (mono-species) and will be denoted by $J_{i}^{m E}$. The second term in the same expression describes elastic collisions between particles of different species (bi-species) and will be denoted by $J_{i}^{b E}$. The last term singles out the fraction $\beta_{i j}$ of those pre-collisional states that are energetic enough to result in chemical reaction, and thus prevent double counting of these collisions in the elastic and reactive operators. This term will be denoted by $J_{i j}^{b * E}$. Accordingly, in what follows, for $i=1,2,3,4$, we will write

$$
J_{i}^{E}=J_{i}^{m E}+J_{i}^{b E}-J_{i j}^{b * E},
$$

with the following notations

$$
\begin{aligned}
J_{i}^{m E} & =\sigma_{i i}^{2} \int_{\mathbb{R}^{3}} \int_{\mathbb{S}_{+}^{2}}\left[f_{i}{ }^{\prime} f_{i_{*}}^{\prime}-f_{i} f_{i_{*}}\right]\left\langle\epsilon, \mathbf{v}_{i}-\mathbf{v}_{i_{*}}\right\rangle d \epsilon d \mathbf{v}_{i_{*}}, \\
J_{i}^{b E} & =\sum_{\substack{s=1 \\
s \neq i}}^{4} \underbrace{\sigma_{i s}^{2} \int_{\mathbb{R}^{3}} \int_{\mathbb{S}_{+}^{2}}\left[f_{i}{ }^{\prime} f_{s}{ }^{\prime}-f_{i} f_{s}\right]\left\langle\epsilon, \mathbf{v}_{i}-\mathbf{v}_{s}\right\rangle d \epsilon d \mathbf{v}_{s}}_{\mathcal{Q}_{i s}}, \\
J_{i}^{b * E} & =\beta_{i j} \sigma_{i j}^{2} \int_{\mathbb{R}^{3}} \int_{\mathbb{S}_{+}^{2}}\left[f_{i}{ }^{\prime} f_{j}{ }^{\prime}-f_{i} f_{j}\right] \Theta\left(\left\langle\epsilon, \mathbf{v}_{i}-\mathbf{v}_{j}\right\rangle-\Xi_{i j}\right)\left\langle\epsilon, \mathbf{v}_{i}-\mathbf{v}_{j}\right\rangle d \epsilon d \mathbf{v}_{j} .
\end{aligned}
$$

The following proposition provides an alternative form of writing the collision operator $J_{i}$, which is very useful to interpret the collisional dynamics of the model, in particular, the role of the operator $J_{i j}^{b * E}$. For the proof, it is enough to combine $J_{i j}^{b * E}$ with the contribution from $J_{i}^{b E}$ when $s=j$ and use the identity $\Theta(x)+\Theta(-x)=1$.

Proposition 3.5 For each $i=1,2,3,4$, the collision operator $J_{i}$ introduced in 28 can be rewritten as

$$
J_{i}=\sigma_{i i}^{2} \int_{\mathbb{R}^{3}} \int_{\mathbb{S}_{+}^{2}}\left[f_{i}^{\prime} f_{i_{*}}^{\prime}-f_{i} f_{i_{*}}\right]\left\langle\epsilon, \mathbf{v}_{i}-\mathbf{v}_{i_{*}}\right\rangle d \epsilon d \mathbf{v}_{i_{*}}
$$




$$
\begin{aligned}
& +\sigma_{i k}^{2} \int_{\mathbb{R}^{3}} \int_{\mathbb{S}_{+}^{2}}\left[f_{i}{ }^{\prime} f_{k}^{\prime}-f_{i} f_{k}\right]\left\langle\epsilon, \mathbf{v}_{i}-\mathbf{v}_{k}\right\rangle d \epsilon d \mathbf{v}_{k}+\sigma_{i l}^{2} \int_{\mathbb{R}^{3}} \int_{\mathbb{S}_{+}^{2}}\left[f_{i}^{\prime} f_{l}^{\prime}-f_{i} f_{l}\right]\left\langle\epsilon, \mathbf{v}_{i}-\mathbf{v}_{l}\right\rangle d \epsilon d \mathbf{v}_{l} \\
& +\sigma_{i j}^{2} \int_{\mathbb{R}^{3}} \int_{\mathbb{S}_{+}^{2}} \Theta\left(\Xi_{i j}-\left\langle\epsilon, \mathbf{v}_{i}-\mathbf{v}_{j}\right\rangle\right)\left[f_{i}{ }^{\prime} f_{j}{ }^{\prime}-f_{i} f_{j}\right]\left\langle\epsilon, \mathbf{v}_{i}-\mathbf{v}_{j}\right\rangle d \epsilon d \mathbf{v}_{j} \\
& +\left(1-\beta_{i j}\right) \sigma_{i j}^{2} \int_{\mathbb{R}^{3}} \int_{\mathbb{S}_{+}^{2}} \Theta\left(\left\langle\epsilon, \mathbf{v}_{i}-\mathbf{v}_{j}\right\rangle-\Xi_{i j}\right)\left[f_{i}{ }^{\prime} f_{j}{ }^{\prime}-f_{i} f_{j}\right]\left\langle\epsilon, \mathbf{v}_{i}-\mathbf{v}_{j}\right\rangle d \epsilon d \mathbf{v}_{j} \\
& +\beta_{i j} \sigma_{i j}^{2} \int_{\mathbb{R}^{3}} \int_{\mathbb{S}_{+}^{2}}\left[\left(\frac{\mu_{i j}}{\mu_{k l}}\right)^{2} f_{k}^{\circ} f_{l}^{\circ}-f_{i} f_{j}\right] \Theta\left(\left\langle\epsilon, \mathbf{v}_{i}-\mathbf{v}_{j}\right\rangle-\Xi_{i j}\right)\left\langle\epsilon, \mathbf{v}_{i}-\mathbf{v}_{j}\right\rangle d \epsilon d \mathbf{v}_{j} .
\end{aligned}
$$

Let us focus on the last three terms on the right hand side of expression $(36)$, which are associated to collisions between the reactive species $A_{i}$ and $A_{j}$. The first of these terms, with $\sigma_{i j}^{2}$ in front of it, is related to those collisions between $A_{i}, A_{j}$ with insufficient amount of energy to produce a chemical reaction, and therefore are governed by elastic collisional dynamics. The last two terms correspond to collisions between $A_{i}, A_{j}$ with sufficient amount of energy to produce a chemical reaction. However, only a fraction $\beta_{i j}$ of such collisions results in a chemical reaction (last term) and produces species $A_{k}$ and $A_{l}$. The remaining fraction $\left(1-\beta_{i j}\right)$ corresponds to collisions that are also governed by elastic collisional dynamics (second to the last term).

Remark 3.6 Observe that by setting the coefficients $\beta_{i j}$ equal to zero, the collisional terms $J_{i}^{b * E}$ and $J_{i}^{R}$ vanish, see (30) and (35). This corresponds to a situation in which the chemical reaction is turned off and we recover from our equations the hard-spheres model for a non-reactive mixture. Moreover, by setting the coefficients equal to one, all collisions with sufficient amount of energy to produce a chemical reaction will result, in fact, in a reactive collision. However, this is not the case in general, because it is well known in chemistry [41] that besides the activation energy barrier, the relative orientation of the molecules at the instant of collision is very important for the occurrence of a chemical reaction, meaning that only collisions with sufficient amount of energy and right orientation will result in a chemical reaction. Accordingly, we will consider in this paper the case in which $\left.\beta_{i j} \in\right] 0,1\left[\right.$ to guarantee that chemical reaction in fact occurs $\left(\beta_{i j}>0\right)$, but some collisions between the reactive species will not result in a chemical reaction due to improper orientation $\left(\beta_{i j}<1\right)$, even if they have enough energy to react chemically.

\subsection{Fundamental Properties of The SRS Model}

In this subsection we review some fundamental properties of the SRS kinetic system. We have decided to include these properties in our paper because here we split the elastic collision operator in a particular form, see (32). These properties are adapted to our formalism and can be proved adapting the proofs in [14, 20, 29, 30, for similar results.

Lemma 3.7 Given the mono-species elastic collision operator $J_{i}^{m E}$, let $\varphi\left(\mathbf{v}_{i}\right)$ be a sufficiently smooth test function. Then, the weak form of (33) for each of the species in the reactive mixture is given by

$$
\begin{aligned}
\int_{\mathbb{R}^{3}} J_{i}^{m E} \varphi\left(\mathbf{v}_{i}\right) d \mathbf{v}_{i}=\frac{1}{4} \sigma_{i i}^{2} \int_{\mathbb{R}^{3}} \int_{\mathbb{R}^{3}} \int_{\mathbb{S}_{+}^{2}}\left[f_{i}^{\prime} f_{i *}^{\prime}-f_{i} f_{i *}\right]\left\langle\epsilon, \mathbf{v}_{i}-\mathbf{v}_{i *}\right\rangle \\
\times\left[\varphi\left(\mathbf{v}_{i}\right)+\varphi\left(\mathbf{v}_{i *}\right)-\varphi\left(\mathbf{v}_{i}^{\prime}\right)-\varphi\left(\mathbf{v}_{i *}^{\prime}\right)\right] d \epsilon d \mathbf{v}_{i *} d \mathbf{v}_{i} .
\end{aligned}
$$

Lemma 3.8 Given the bi-species elastic collision operator $J_{i}^{b E}$ defined in (34) as a sum of several contributions of $\mathcal{Q}_{i s}$, let $\varphi\left(\mathbf{v}_{i}\right)$ be a sufficiently smooth test function. Then, for each $i, s=1,2,3,4$ with $i \neq s$, we have that

$$
\int_{\mathbb{R}^{3}} \mathcal{Q}_{i s} \varphi\left(\mathbf{v}_{i}\right) d \mathbf{v}_{i}=\sigma_{i s}^{2} \int_{\mathbb{R}^{3}} \int_{\mathbb{R}^{3}} \int_{\mathbb{S}_{+}^{2}}\left[\varphi\left(\mathbf{v}_{i}^{\prime}\right)-\varphi\left(\mathbf{v}_{i}\right)\right] f_{i} f_{s}\left\langle\epsilon, \mathbf{v}_{i}-\mathbf{v}_{s}\right\rangle d \epsilon d \mathbf{v}_{s} d \mathbf{v}_{i}
$$


Lemma 3.9 Given the elastic collision operator $J_{i j}^{b * E}$ defined in $(35)$, let $\varphi\left(\mathbf{v}_{i}\right)$ be a sufficiently smooth test function. If we assume that $\beta_{i j}=\beta_{j i}$, then for $(i, j) \in\{(1,2),(2,1),(3,4),(4,3)\}$, we have that

$$
\begin{aligned}
& \int_{\mathbb{R}^{3}} J_{i j}^{b * E} \varphi\left(\mathbf{v}_{i}\right) d \mathbf{v}_{i}=\beta_{i j} \sigma_{i j}^{2} \int_{\mathbb{R}^{3}} \int_{\mathbb{R}^{3}} \int_{\mathbb{S}_{+}^{2}}\left[\varphi\left(\mathbf{v}_{i}^{\prime}\right)-\varphi\left(\mathbf{v}_{i}\right)\right] f_{i} f_{j} \Theta\left(\left\langle\epsilon, \mathbf{v}_{i}-\mathbf{v}_{j}\right\rangle-\Xi_{i j}\right) \\
& \times\left\langle\epsilon, \mathbf{v}_{i}-\mathbf{v}_{j}\right\rangle d \epsilon d \mathbf{v}_{j} d \mathbf{v}_{i} .
\end{aligned}
$$

Concerning the reactive collision operator $J_{i}^{R}$, we have the following property.

Lemma 3.10 Given the reactive collision operator $J_{i}^{R}$ defined in $(30)$, let $\varphi\left(\mathbf{v}_{i}\right)$ be a sufficiently smooth test function. If we assume that $\beta_{i j}=\beta_{j i}$ and $\beta_{12} \sigma_{12}^{2}=\beta_{34} \sigma_{34}^{2}$, then we have that

$$
\begin{array}{r}
\sum_{i=1}^{4} \int_{\mathbb{R}^{3}} J_{i}^{R} \varphi_{i}\left(\mathbf{v}_{i}\right) d \mathbf{v}_{i}=\beta_{12} \sigma_{12}^{2} \int_{\mathbb{R}^{3}} \int_{\mathbb{R}^{3}} \int_{\mathbb{S}_{+}^{2}}\left[\varphi_{1}+\varphi_{2}-\varphi_{3}^{\circ}-\varphi_{4}^{\circ}\right]\left[\left(\frac{\mu_{12}}{\mu_{34}}\right)^{2} f_{3}^{\circ} f_{4}^{\circ}-f_{1} f_{2}\right] \\
\times \Theta\left(\left\langle\epsilon, \mathbf{v}_{1}-\mathbf{v}_{2}\right\rangle-\Xi_{12}\right)\left\langle\epsilon, \mathbf{v}_{1}-\mathbf{v}_{2}\right\rangle d \epsilon d \mathbf{v}_{2} d \mathbf{v}_{1} \\
=\beta_{34} \sigma_{34}^{2} \int_{\mathbb{R}^{3}} \int_{\mathbb{R}^{3}} \int_{\mathbb{S}_{+}^{2}}\left[\varphi_{3}+\varphi_{4}-\varphi_{1}^{\circ}-\varphi_{2}^{\circ}\right]\left[\left(\frac{\mu_{34}}{\mu_{12}}\right)^{2} f_{1}^{\circ} f_{2}^{\circ}-f_{3} f_{4}\right] \\
\times \Theta\left(\left\langle\epsilon, \mathbf{v}_{3}-\mathbf{v}_{4}\right\rangle-\Xi_{34}\right)\left\langle\epsilon, \mathbf{v}_{3}-\mathbf{v}_{4}\right\rangle d \epsilon d \mathbf{v}_{4} d \mathbf{v}_{3} .
\end{array}
$$

\subsection{Conservation equations}

The conservation equations of the SRS model are obtained from the properties stated in Subsection 3.3 . Their proofs are rather standard and follow the same line as in [14, 20, 29, 30].

Corollary 3.11 The mono-species elastic collision operator given in $(33)$ is such that, for $i=1,2,3,4$,

$$
\int_{\mathbb{R}^{3}} J_{i}^{m E}\left(\begin{array}{c}
1 \\
m_{i} \mathbf{v}_{i} \\
\frac{1}{2} m_{i}\left(\mathbf{v}_{i}\right)^{2}
\end{array}\right) d \mathbf{v}_{i}=0 .
$$

Proof. The proof follows from Lemma 3.7 and conservation laws 13 .

Corollary 3.12 Let $\mathcal{Q}_{i s}$ and $J_{i j}^{b * E}$ be as defined in (34) and (35), respectively. Then

$$
\begin{aligned}
& \int_{\mathbb{R}^{3}} \mathcal{Q}_{i s} d \mathbf{v}_{i}=0, \\
& \int_{\mathbb{R}^{3}} J_{i j}^{b * E} d \mathbf{v}_{i}=0, \\
& \int_{\mathbb{R}^{3}} \mathcal{Q}_{i s}\left(\begin{array}{c}
m_{i} \mathbf{v}_{i} \\
\frac{1}{2} m_{i}\left(\mathbf{v}_{i}\right)^{2}
\end{array}\right) d \mathbf{v}_{i}+\int_{\mathbb{R}^{3}} \mathcal{Q}_{s i}\left(\begin{array}{c}
m_{s} \mathbf{v}_{s} \\
\frac{1}{2} m_{s}\left(\mathbf{v}_{s}\right)^{2}
\end{array}\right) d \mathbf{v}_{s}=0, \\
& \int_{\mathbb{R}^{3}} J_{i j}^{b * E}\left(\begin{array}{c}
m_{i} \mathbf{v}_{i} \\
\frac{1}{2} m_{i}\left(\mathbf{v}_{i}\right)^{2}
\end{array}\right) d \mathbf{v}_{i}+\int_{\mathbb{R}^{3}} J_{j i}^{b * E}\left(\begin{array}{c}
m_{j} \mathbf{v}_{j} \\
\frac{1}{2} m_{j}\left(\mathbf{v}_{j}\right)^{2}
\end{array}\right) d \mathbf{v}_{j}=0 .
\end{aligned}
$$

Proof. The proof follows from Lemmas 3.8, 3.9 and conservation laws 13. 
Corollary 3.13 The reactive collision operators satisfy the following property

$$
\int_{\mathbb{R}^{3}} J_{1}^{R} d \mathbf{v}_{1}=\int_{\mathbb{R}^{3}} J_{2}^{R} d \mathbf{v}_{2}=-\int_{\mathbb{R}^{3}} J_{3}^{R} d \mathbf{v}_{3}=-\int_{\mathbb{R}^{3}} J_{4}^{R} d \mathbf{v}_{4} .
$$

Proof. The proof follows from Lemma 3.10 .

Corollary 3.13 assures the correct exchange rates for the species in the chemical reaction (1).

Corollary 3.14 The elastic and reactive collision operators are such that

$$
\sum_{i=1}^{4} \int_{\mathbb{R}^{3}} \varphi\left(\mathbf{v}_{i}\right)\left(J_{i}^{E}+J_{i}^{R}\right) d \mathbf{v}_{i}=0
$$

with $\varphi\left(\mathbf{v}_{i}\right)$ alternatively given by $\varphi\left(\mathbf{v}_{i}\right)=(1,0,1,0), \varphi\left(\mathbf{v}_{i}\right)=(1,0,0,1), \varphi\left(\mathbf{v}_{i}\right)=(0,1,1,0)$, or by $\varphi\left(\mathbf{v}_{i}\right)=m_{i} v_{i x}$, $\varphi\left(\mathbf{v}_{i}\right)=m_{i} v_{i y}, \varphi\left(\mathbf{v}_{i}\right)=m_{i} v_{i z}$, or by $\varphi\left(\mathbf{v}_{i}\right)=E_{i}+\frac{1}{2} m_{i} \mathbf{v}_{i}^{2}$, where $v_{i x}$, $v_{i y}$ and $v_{i z}$ represent the spatial components of the molecular velocity $\mathbf{v}_{i}$.

Proof. The proof follows from Corollaries $3.11,3.12$ and Lemma 3.10 .

Corollary 3.14 indicates that, at least formally, the SRS model possesses seven independent macroscopic conservation laws, for the total number of particles of the reactant-product pairs of the form $A_{1}-A_{3}, A_{1}-A_{4}$ and $A_{2}-A_{3}$, the three momentum components and the total energy of the mixture.

\subsection{Equilibrium solutions and H-Theorem}

The equilibrium solutions of the SRS system are characterized as follows.

Definition 3.15 The equilibrium solutions of the SRS model 28) are distribution functions $f_{i}\left(t, \mathbf{x}, \mathbf{v}_{i}\right)$ such that the operators $J_{i}^{E}$ and $J_{i}^{R}$ given in (29) and (30) satisfy

$$
J_{i}^{E}+J_{i}^{R}=0, \quad i=1, \ldots, 4 .
$$

Proposition 3.16 If the coefficients $\beta_{i j}$ and the reactive cross sections are such that $\beta_{i j}=\beta_{j i}$ and $\beta_{12} \sigma_{12}^{2}=$ $\beta_{34} \sigma_{34}^{2}$, then the following statements are equivalent

(a) $f_{i}=c_{i}\left(\frac{m_{i}}{2 \pi k_{B} T}\right)^{3 / 2} \exp \left(-\frac{m_{i}\left(\mathbf{v}_{i}-\mathbf{u}\right)^{2}}{2 k_{B} T}\right)$, for $i=1, \ldots, 4$, with

$$
c_{1} c_{2}=\left(\frac{\mu_{12}}{\mu_{34}}\right)^{1 / 2} c_{3} c_{4} \exp \left(\frac{Q_{R}}{k_{B} T}\right)
$$

(b) $J_{i}^{E}=0$ and $J_{i}^{R}=0, \quad i=1, \ldots, 4$;

(c) $\sum_{i=1}^{4} \int_{\mathbb{R}^{3}}\left[J_{i}^{E}+J_{i}^{R}\right] \log \left(\frac{f_{i}}{\mu_{i j}}\right) d v=0$,

where

$$
c_{i}(t, \mathbf{x})=\int_{\mathbb{R}^{3}} f_{i}\left(t, \mathbf{x}, \mathbf{v}_{i}\right) d \mathbf{v}_{i}, \quad i=1, \ldots, 4
$$




$$
\begin{aligned}
& \mathbf{u}(t, \mathbf{x})=\sum_{i=1}^{4} \int_{\mathbb{R}^{3}} m_{i} \mathbf{v}_{i} f_{i}\left(t, \mathbf{x}, \mathbf{v}_{i}\right) d \mathbf{v}_{i} / \sum_{i=1}^{4} \int_{\mathbb{R}^{3}} m_{i} f_{i}\left(t, \mathbf{x}, \mathbf{v}_{i}\right) d \mathbf{v}_{i}, \\
& T(t, \mathbf{x})=\frac{1}{3 k_{B}} \sum_{i=1}^{4} \int_{\mathbb{R}^{3}} m_{i}\left(\mathbf{v}_{i}-\mathbf{u}\right)^{2} f_{i}\left(t, \mathbf{x}, \mathbf{v}_{i}\right) d \mathbf{v}_{i} / \sum_{i=1}^{4} c_{i}(t, \mathbf{x}) .
\end{aligned}
$$

Condition (49) represents the so called mass action law for the SRS kinetic model.

\section{Reaction diffusion limit of the SRS kinetic model}

In this section we formally derive the reaction-diffusion system of MS type as a hydrodynamic limit of the SRS kinetic model given in Section 3. In order to achieve this, we have to define an evolution regime for the chemical process and consider the mathematical assumptions that should be imposed on the kinetic model in agreement with the physical conditions associated to the MS setting.

\subsection{The scaled equations and our assumptions}

The starting point for the derivation of the reaction diffusion system of MS type is the scaled SRS kinetic system in a form compatible with the considered chemical regime of dominant elastic collisions and slow chemical reactions. Accordingly, we will consider the SRS kinetic equations written in non-dimensional form as

$$
S t \frac{\partial \widehat{f}_{i}}{\partial \widehat{t}}+\widehat{\mathbf{v}}_{i} \cdot \frac{\partial \widehat{f}_{i}}{\partial \widehat{\mathbf{x}}}=\frac{1}{K n_{e}} \widehat{J}_{i}^{m E}+\frac{1}{K n_{e}} \widehat{J}_{i}^{b E}-\frac{1}{K n_{r}} \widehat{J}_{i j}^{b * E}+\frac{1}{K n_{r}} \widehat{J}_{i}^{R}, \quad i=1,2,3,4,
$$

where $S t$ is the kinetic Strouhal number [19, $K n_{e}$ and $K n_{r}$ are the elastic and reactive Knudsen numbers [1. The Knudsen numbers are such that the reactive and elastic mean free paths are related by the factor $K n_{r} / K n_{e}=1 / \beta_{i j}$. The symbols with the hat indicate scaled quantities with respect to a reference length $L$, time $\tau$ and temperature $T_{0}$. We also introduce the speed of sound $c_{0}$ in a mixture of monatomic ideal gases

at temperature $T_{0}$, given by $c_{0}=\sqrt{\frac{5 n_{0} k_{B} T_{0}}{3 \rho_{0}}}$, and scale the velocities with respect to $c_{0}$. The scaled collisional operators are defined by

$$
\begin{aligned}
& \widehat{J}_{i}^{m E}=\widehat{\sigma}_{i i}^{2} \int_{\mathbb{R}^{3}} \int_{\mathbb{S}_{+}^{2}}\left[\widehat{f}_{i}^{\prime} \widehat{f}_{i *}^{\prime}-\widehat{f}_{i} \widehat{f}_{i *}\right]\left\langle\epsilon, \widehat{\mathbf{v}}_{i}-\widehat{\mathbf{v}}_{i *}\right\rangle d \epsilon d \widehat{\mathbf{v}}_{i *}, \\
& \widehat{J}_{i}^{b E}=\sum_{\substack{s=1 \\
s \neq i}}^{4} \widehat{\sigma}_{i s}^{2} \int_{\mathbb{R}^{3}} \int_{\mathbb{S}_{+}^{2}}\left[\widehat{f}_{i}^{\prime} \widehat{f}_{s}^{\prime}-\widehat{f}_{i} \widehat{f}_{s}\right]\left\langle\epsilon, \widehat{\mathbf{v}}_{i}-\widehat{\mathbf{v}}_{s}\right\rangle d \epsilon d \widehat{\mathbf{v}}_{s}, \\
& \widehat{J}_{i j}^{b * E}=\widehat{\sigma}_{i j}^{2} \int_{\mathbb{R}^{3}} \int_{\mathbb{S}_{+}^{2}}\left[\widehat{f}_{i}^{\prime} \widehat{f}_{j}^{\prime}-\widehat{f}_{i} \widehat{f}_{j}\right] \Theta\left(\left\langle\epsilon, c_{0} \widehat{\mathbf{v}}_{i}-c_{0} \widehat{\mathbf{v}}_{j}\right\rangle-\Xi_{i j}\right)\left\langle\epsilon, \widehat{\mathbf{v}}_{i}-\widehat{\mathbf{v}}_{j}\right\rangle d \epsilon d \widehat{\mathbf{v}}_{j}, \\
& \widehat{J}_{i}^{R}=\widehat{\sigma}_{i j}^{2} \int_{\mathbb{R}^{3}} \int_{\mathbb{S}_{+}^{2}}\left[\left(\frac{\mu_{i j}}{\mu_{k l}}\right)^{2} \widehat{f}_{k}^{\circ} \widehat{f}_{l}^{\circ}-\widehat{f}_{i} \widehat{f}_{j}\right] \Theta\left(\left\langle\epsilon, c_{0} \widehat{\mathbf{v}}_{i}-c_{0} \widehat{\mathbf{v}}_{j}\right\rangle-\Xi_{i j}\right)\left\langle\epsilon, \widehat{\mathbf{v}}_{i}-\widehat{\mathbf{v}}_{j}\right\rangle d \epsilon d \widehat{\mathbf{v}}_{j} .
\end{aligned}
$$

Moreover, we assume that the bulk velocity of the mixture is comparable to $L / \tau$ and, as a result, the Strouhal number becomes the Mach number, $M a$. Henceforth, we introduce the notations

$$
S t=M a=\alpha, \quad K n_{e}=\alpha^{p} \quad \text { and } \quad K n_{r}=\alpha^{q},
$$

where $\alpha \ll 1, p$ and $q$ are real numbers with $p \geq 1$ and $p>q$. Also, we assume that the elastic Knudsen number is of the same order of magnitude as the Mach number, so that $p=1$. Additionally, we are interested in a chemical regime for which elastic collisions are predominant and reactive collisions are rare, so that we assume 
$q=-1$. Using the above notations and the generic assumptions in [51, and removing the hats for simplicity, we obtain that, for $i=1,2,3,4$,

$$
\alpha \frac{\partial f_{i}^{\alpha}}{\partial t}+\mathbf{v}_{i} \cdot \frac{\partial f_{i}^{\alpha}}{\partial \mathbf{x}}=\frac{1}{\alpha} J_{i}^{m \alpha E}+\frac{1}{\alpha} J_{i}^{b \alpha E}-\alpha J_{i j}^{b * \alpha E}+\alpha J_{i}^{\alpha R}, \quad \text { in } \quad \mathbb{R}_{+} \times \Omega \times \mathbb{R}^{3},
$$

where $f_{i}^{\alpha}, i=1,2,3,4$, are now the scaled unknowns in the considered regime and $J_{i}^{m \alpha E}, J_{i}^{b \alpha E}, J_{i j}^{b * \alpha E}, J_{i}^{\alpha R}$ indicate that the collisional operators are now expressed in terms of $f_{i}^{\alpha}$. Equations (53) emphasize that the mono-species and bi-species elastic operators $J_{i}^{m \alpha E}$ and $J_{i}^{b \alpha E}$ are associated to predominant effects, whereas the reactive operator $J_{i}^{\alpha R}$ and the correction elastic term $J_{i j}^{b * \alpha E}$ are associated to rare effects.

With the obvious adjustments, the conservation laws and properties of the SRS model given in Section 3 are still valid for the scaled equations (53).

Besides the chemical regime of slow chemical reaction, our central assumptions to derive the reaction diffusion system of MS type from the kinetic formulation are as follows:

(a) The initial conditions are local Maxwellian functions centred at the mean velocity of the species.

(b) The evolution of the system leaves the distribution functions in the local Max-wellian state.

(c) The bulk velocity of the mixture is small and goes to zero as $\alpha \rightarrow 0$.

(d) The evolution of the mixture obeys the isothermal condition.

Assumption (d) implies that the temperature $T$ of the mixture is uniform in space and constant in time. It is compatible with the physical situation of slow chemical reaction and signifies that the chemical process takes place at a rate slow enough to allow the surroundings to continuously compensate for the balance of reaction heat between the reactants and products (see 43). Assumption (c) allows us to neglect convective effects and describe a physical system governed by diffusion and chemical reaction. Assumptions (a) and (b) correspond to the physical situation in which the system evolves not far away from the local Maxwellian equilibrium.

From both the assumption (a) and the first assertion of (52), and following [18, 19, the initial conditions for (53) are distribution functions of the following form

$$
f_{i}^{\alpha(i n)}\left(\mathbf{x}, \mathbf{v}_{i}\right)=c_{i}^{\alpha(i n)}(\mathbf{x})\left(\frac{m_{i}}{2 \pi k_{B} T}\right)^{\frac{3}{2}} \exp \left[-\frac{m_{i}\left(\mathbf{v}_{i}-\alpha \mathbf{u}_{i}^{\alpha(i n)}(\mathbf{x})\right)^{2}}{2 k_{B} T}\right], \mathbf{x} \in \Omega, \mathbf{v}_{i} \in \mathbb{R}^{3}
$$

where $T>0$ is constant (isothermal condition), $c_{i}^{\alpha(i n)}: \Omega \rightarrow \mathbb{R}_{+}$, for $i=1, \ldots, 4$, with $c^{\alpha(i n)}=\sum_{i=1}^{4} c_{i}^{\alpha(i n)}$, and $\mathbf{u}_{i}^{\alpha(i n)}: \Omega \rightarrow \mathbb{R}^{3}$, for $i=1, \ldots, 4$. From assumption (b), the distribution functions at time $t>0$ are local Maxwellians centred at the mean velocity of the species, given by

$$
f_{i}^{\alpha}\left(t, \mathbf{x}, \mathbf{v}_{i}\right)=c_{i}^{\alpha}(t, \mathbf{x})\left(\frac{m_{i}}{2 \pi k_{B} T}\right)^{\frac{3}{2}} \exp \left[-\frac{m_{i}\left(\mathbf{v}_{i}-\alpha \mathbf{u}_{i}^{\alpha}(t, \mathbf{x})\right)^{2}}{2 k_{B} T}\right], \quad \mathbf{x} \in \Omega, \mathbf{v}_{i} \in \mathbb{R}^{3},
$$

where $c_{i}^{\alpha}: \mathbb{R}_{+} \times \Omega \rightarrow \mathbb{R}_{+}$and $\mathbf{u}_{i}^{\alpha}: \mathbb{R}_{+} \times \Omega \rightarrow \mathbb{R}^{3}$, for $i=1, \ldots 4$. Using (55), the moments of order 0 and 1 of the distribution functions are, respectively, given by

$$
\int_{\mathbb{R}^{3}} f_{i}^{\alpha}\left(t, \mathbf{x}, \mathbf{v}_{i}\right) d \mathbf{v}_{i}=c_{i}^{\alpha}(t, \mathbf{x}) \quad \text { and } \quad \int_{\mathbb{R}^{3}} \mathbf{v}_{i} f_{i}^{\alpha}\left(t, \mathbf{x}, \mathbf{v}_{i}\right) d \mathbf{v}_{i}=\alpha c_{i}^{\alpha}(t, \mathbf{x}) \mathbf{u}_{i}^{\alpha}(t, \mathbf{x}) .
$$

An important step in the passage from a kinetic model to hydrodynamic equations is the explicit computation of the integral contributions appearing in the kinetic equations. When a reactive mixture is involved, such computation can be very intricate, essentially because of the redistribution of masses and internal energies 
among the constituents. Having this in mind, in the light of assumption (c), the Maxwellian (55) with species velocity will be approximated through a first order expansion around the Maxwellian $M_{i}^{\alpha}$ with a common and vanishing mixture velocity, namely

$$
f_{i}^{\alpha}\left(t, \mathbf{x}, \mathbf{v}_{i}\right) \approx M_{i}^{\alpha}\left(t, \mathbf{x}, \mathbf{v}_{i}\right)\left[1+\frac{\alpha m_{i} \mathbf{v}_{\mathbf{i}} \cdot \mathbf{u}_{\mathbf{i}}^{\alpha}(\mathbf{t}, \mathbf{x})}{k_{B} T}\right]
$$

with

$$
M_{i}^{\alpha}\left(t, \mathbf{x}, \mathbf{v}_{i}\right)=c_{i}^{\alpha}(t, \mathbf{x})\left(\frac{m_{i}}{2 \pi k_{B} T}\right)^{\frac{3}{2}} \exp \left(-\frac{m_{i}\left(\mathbf{v}_{i}\right)^{2}}{2 k_{B} T}\right) .
$$

Expressions (57) will be used in Subsections 4.2 and 4.3 for the explicit computation of the integral contributions. As stated by Present in [31, p. 147, first-order corrections to the Maxwellian distributions (58) as those given by expansions (57) were first used by J. Stefan in his celebrated work from 1872 to capture the diffusion effects in a mixture of gases.

Moreover, hereinafter we will use the notation $\Gamma(\eta, x)$ for the incomplete Euler gamma function defined by

$$
\Gamma(\eta, x)=\int_{x}^{\infty} t^{\eta-1} e^{-t} d t
$$

\subsection{Concentration balance equations}

The balance equations for the species concentration can formally be derived from the scaled SRS equations given in (53), by integrating over the velocity space. Also, the conservation laws and some of the fundamental properties of the kinetic model stated in Section 3 will be used to achieve the following result.

Lemma 4.1 The concentration balance equations for the species in the reactive mixture can be written as

$$
\frac{\partial c_{i}^{\alpha}}{\partial t}+\frac{\partial}{\partial \mathbf{x}}\left(c_{i}^{\alpha} \mathbf{u}_{i}^{\alpha}\right)=\mathcal{J}_{i}, \quad i=1, \ldots, 4,
$$

with $\mathcal{J}_{i}$ being the reaction rate of the $i$-th species given by

$$
\begin{aligned}
\mathcal{J}_{i}=\sigma_{i j}^{2}\left(\frac{2 \pi \mu_{i j}}{k_{B} T}\right)^{\frac{1}{2}} & {\left[\left(\frac{\mu_{i j}}{\mu_{k l}}\right)^{\frac{1}{2}} c_{k}^{\alpha} c_{l}^{\alpha} \exp \left(\frac{Q_{R}}{k_{B} T}\right)-c_{i}^{\alpha} c_{j}^{\alpha}\right] } \\
& \times\left[\frac{2 k_{B} T}{\mu_{i j}} \Gamma\left(2, z_{i}^{*}\right)-\left(\frac{\Xi_{i j}}{c_{0}}\right)^{2} \Gamma\left(1, z_{i}^{*}\right)\right],
\end{aligned}
$$

where the terms $\Gamma\left(1, z_{i}^{*}\right), \Gamma\left(2, z_{i}^{*}\right)$ are defined by $[59$ with

$$
z_{i}^{*}=\frac{\mu_{i j}}{2 k_{B} T}\left(\frac{\Xi_{i j}}{c_{0}}\right)^{2} \text {. }
$$

Moreover, for each $i=1, \ldots, 4$ we have $\mathcal{J}_{i}=\lambda_{i} \mathcal{J}_{1}$, with $\lambda_{1}=\lambda_{2}=1$ and $\lambda_{3}=\lambda_{4}=-1$.

Proof. First, we integrate both sides of the scaled SRS equations given in 53 with respect to $\mathbf{v}_{i} \in \mathbb{R}^{3}$, to obtain

$$
\alpha \frac{\partial}{\partial t}\left(\int_{\mathbb{R}^{3}} f_{i}^{\alpha} d \mathbf{v}_{i}\right)+\frac{\partial}{\partial \mathbf{x}}\left(\int_{\mathbb{R}^{3}} \mathbf{v}_{i} f_{i}^{\alpha} d \mathbf{v}_{i}\right)=\alpha \int_{\mathbb{R}^{3}} J_{i}^{\alpha R} d \mathbf{v}_{i}
$$

where we have used Corollary 3.11. Lemma 3.8 with $\varphi\left(\mathbf{v}_{i}\right)=1$, and Lemma 3.9 with $\varphi\left(\mathbf{v}_{i}\right)=1$. Using [56), we obtain

$$
\frac{\partial c_{i}^{\alpha}}{\partial t}+\frac{\partial}{\partial \mathbf{x}}\left(c_{i}^{\alpha} \mathbf{u}_{i}^{\alpha}\right)=\int_{\mathbb{R}^{3}} J_{i}^{\alpha R} d \mathbf{v}_{i}
$$


Next, we derive an explicit expression for the integral contribution on the right hand side of (63). To do this, we replace the distribution functions by their approximations (57). For sake of simplicity, we write such approximations in the form

$$
f_{i}^{\alpha} \approx M_{i}^{\alpha}\left(1+\mathbf{a}_{i} \cdot \mathbf{v}_{i}\right), \quad \text { with } \quad \mathbf{a}_{i}=\frac{\alpha m_{i} \mathbf{u}_{i}^{\alpha}}{k_{B} T}
$$

and, for any index $i$, we refer to $\mathbf{a}_{i}$ as coefficient $\mathbf{a}$. Using (64), we can write the integral on the right-hand-side of (63) as the sum of the following three terms,

$$
\begin{aligned}
& \mathcal{D}=\sigma_{i j}^{2} \int_{\mathbb{R}^{3}} \int_{\mathbb{R}^{3}} \int_{\mathbb{S}_{+}^{2}}\left(\frac{\mu_{i j}^{2}}{\mu_{k l}^{2}} M_{k}^{\alpha \circ} M_{l}^{\alpha \circ}-M_{i}^{\alpha} M_{j}^{\alpha}\right) \Theta\left(\left\langle\epsilon, c_{0} \mathbf{v}_{i}-c_{0} \mathbf{v}_{j}\right\rangle-\Xi_{i j}\right)\left\langle\epsilon, \mathbf{v}_{i}-\mathbf{v}_{j}\right\rangle d \epsilon d \mathbf{v}_{j} d \mathbf{v}_{i}, \\
& \mathcal{E}=\sigma_{i j}^{2} \int_{\mathbb{R}^{3}} \int_{\mathbb{R}^{3}} \int_{\mathbb{S}_{+}^{2}}\left(\frac{\mu_{i j}^{2}}{\mu_{k l}^{2}} M_{k}^{\alpha \circ} M_{l}^{\alpha \circ}\left(\mathbf{a}_{k} \cdot \mathbf{v}_{k}^{\circ}+\mathbf{a}_{l} \cdot \mathbf{v}_{l}^{\circ}\right)\right) \Theta\left(\left\langle\epsilon, c_{0} \mathbf{v}_{i}-c_{0} \mathbf{v}_{j}\right\rangle-\Xi_{i j}\right)\left\langle\epsilon, \mathbf{v}_{i}-\mathbf{v}_{j}\right\rangle d \epsilon d \mathbf{v}_{j} d \mathbf{v}_{i}, \\
& \mathcal{F}=-\sigma_{i j}^{2} \int_{\mathbb{R}^{3}} \int_{\mathbb{R}^{3}} \int_{\mathbb{S}_{+}^{2}}\left(M_{i}^{\alpha} M_{j}^{\alpha}\left(\mathbf{a}_{i} \cdot \mathbf{v}_{i}+\mathbf{a}_{j} \cdot \mathbf{v}_{j}\right)\right) \Theta\left(\left\langle\epsilon, c_{0} \mathbf{v}_{i}-c_{0} \mathbf{v}_{j}\right\rangle-\Xi_{i j}\right)\left\langle\epsilon, \mathbf{v}_{i}-\mathbf{v}_{j}\right\rangle d \epsilon d \mathbf{v}_{j} d \mathbf{v}_{i},
\end{aligned}
$$

where quadratic terms in the coefficients a have been neglected.

Inserting (58) into the integral contributions $\mathcal{D}, \mathcal{E}$ and $\mathcal{F}$, we obtain

(i) $\mathcal{D}=\mathcal{J}_{i}$, with $\mathcal{J}_{i}$ the reaction rate defined in 61;

(ii) $\mathcal{E}=0$;

(iii) $\mathcal{F}=0$.

Concerning items (ii) and (iii), the computations require some variable transformations and we give some details in Appendix A of Subsection 6.1. Let us focus on item (i). Using (58) and the conservation law of total energy during reactive collisions given in (18), we obtain

$$
\begin{aligned}
\mathcal{D}= & \sigma_{i j}^{2} \frac{\left(m_{i} m_{j}\right)^{\frac{3}{2}}}{\left(2 \pi k_{B} T\right)^{3}}\left[\left(\frac{\mu_{i j}}{\mu_{k l}}\right)^{\frac{1}{2}} c_{k}^{\alpha} c_{l}^{\alpha} \exp \left(\frac{Q_{R}}{k_{B} T}\right)-c_{i}^{\alpha} c_{j}^{\alpha}\right] \\
& \times \int_{\mathbb{R}^{3}} \int_{\mathbb{R}^{3}} \int_{\mathbb{S}_{+}^{2}} \exp \left[-\frac{m_{i}\left(\mathbf{v}_{i}\right)^{2}+m_{j}\left(\mathbf{v}_{j}\right)^{2}}{2 k_{B} T}\right] \Theta\left(\left\langle\epsilon, c_{0} \mathbf{v}_{i}-c_{0} \mathbf{v}_{j}\right\rangle-\Xi_{i j}\right)\left\langle\epsilon, \mathbf{v}_{i}-\mathbf{v}_{j}\right\rangle d \epsilon d \mathbf{v}_{j} d \mathbf{v}_{i} .
\end{aligned}
$$

Evaluating the integral over $\mathbb{S}_{+}^{2}$, using spherical coordinates as described in Remark 3.1, we obtain

$$
\int_{\mathbb{S}_{+}^{2}} \Theta\left(\left\langle\epsilon, c_{0} \mathbf{v}_{i}-c_{0} \mathbf{v}_{j}\right\rangle-\Xi_{i j}\right)\left\langle\epsilon, \mathbf{v}_{i}-\mathbf{v}_{j}\right\rangle d \epsilon=\pi V \Theta\left(V-\frac{\Xi_{i j}}{c_{0}}\right)\left[1-\left(\frac{\Xi_{i j}}{c_{0} V}\right)^{2}\right] .
$$

Susbstituting (67) into (66), then transforming the resulting sixfold integral in $\mathbf{v}_{i}, \mathbf{v}_{j}$ to the relative velocity $\mathbf{V}=\mathbf{v}_{j}-\mathbf{v}_{i}$ and centre of mass velocity $\mathbf{X}=\left(m_{i} \mathbf{v}_{i}+m_{j} \mathbf{v}_{j}\right) /\left(m_{i}+m_{j}\right)$, and using the fact that the Jacobian of the transformation has absolute value 1 , we get

$$
\begin{aligned}
\mathcal{D}=\pi \sigma_{i j}^{2} \frac{\left(m_{i} m_{j}\right)^{\frac{3}{2}}}{\left(2 \pi k_{B} T\right)^{3}}\left[\left(\frac{\mu_{i j}}{\mu_{k l}}\right)^{\frac{1}{2}} c_{k}^{\alpha} c_{l}^{\alpha} \exp \left(\frac{Q_{R}}{k_{B} T}\right)-c_{i}^{\alpha} c_{j}^{\alpha}\right] \\
\quad \times \int_{\mathbb{R}^{3}} \exp \left(-\frac{M X^{2}}{2 k_{B} T}\right) d \mathbf{X} \int_{\mathbb{R}^{3}} V \Theta\left(V-\frac{\Xi_{i j}}{c_{0}}\right)\left[1-\left(\frac{\Xi_{i j}}{c_{0} V}\right)^{2}\right] \exp \left(-\frac{\mu_{i j} V^{2}}{2 k_{B} T}\right) d \mathbf{V},
\end{aligned}
$$


where $M=m_{i}+m_{j}$. The integral in $\mathbf{X}$ can be easily evaluated and becomes

$$
\int_{\mathbb{R}^{3}} \exp \left(-\frac{M X^{2}}{2 k_{B} T}\right) d \mathbf{X}=\left(\frac{2 \pi k_{B} T}{M}\right)^{\frac{3}{2}}
$$

The integral in $\mathbf{V}$ can be expanded and the resulting terms are computed by first transforming to spherical coordinates. Next, the resulting scalar integrals in $V$ are transformed to $z$, using $z=\frac{\mu_{i j}}{2 k_{B} T} V^{2}$, and finally using (59), we obtain

$$
\begin{aligned}
\int_{\mathbb{R}^{3}} V \Theta\left(V-\frac{\Xi_{i j}}{c_{0}}\right) \exp \left(-\frac{\mu_{i j} V^{2}}{2 k_{B} T}\right) d \mathbf{V}-\int_{\mathbb{R}^{3}} V & \exp \left(-\frac{\mu_{i j} V^{2}}{2 k_{B} T}\right) \Theta\left(V-\frac{\Xi_{i j}}{c_{0}}\right)\left(\frac{\Xi_{i j}}{c_{0} V}\right)^{2} d \mathbf{V} \\
= & 2 \pi \frac{2 k_{B} T}{\mu_{i j}}\left[\frac{2 k_{B} T}{\mu_{i j}} \Gamma\left(2, z_{i}^{*}\right)-\left(\frac{\Xi_{i j}}{c_{0}}\right)^{2} \Gamma\left(1, z_{i}^{*}\right)\right] .
\end{aligned}
$$

Substituting (69) and (70) into (68), and performing a little algebra, we obtain the desired expression (61) for the integral $\mathcal{D}$ in item (i).

The last assertion of Lemma 4.1 is an immediate consequence of Corollary 3.13 about the reactive collision operators.

Remark 4.2 (a) The reaction rate $\mathcal{J}_{i}$ given in (61) can be written in an equivalent form, as a phenomenological law for the chemical reaction (1), as

$$
\mathcal{J}_{i}=-\lambda_{i}\left(k_{f} c_{1}^{\alpha} c_{2}^{\alpha}-k_{b} c_{3}^{\alpha} c_{4}^{\alpha}\right), \quad i=1, \ldots, 4,
$$

with $k_{f}$ and $k_{b}$ being the forward and backward rate constants given, respectively, by

$$
k_{f}=\sigma_{12}^{2} \sqrt{\frac{8 \pi k_{B} T}{\mu_{12}}} \exp \left(-\frac{\zeta_{1}}{k_{B} T}\right) \quad \text { and } \quad k_{b}=\sigma_{34}^{2} \sqrt{\frac{8 \pi k_{B} T}{\mu_{34}}} \exp \left(-\frac{\zeta_{3}}{k_{B} T}\right),
$$

Equation (71) expresses the reaction rate in the form used, in general, in physical applications, see [24].

(b) Our expression (61) differs from the corresponding one obtained in paper [5], see (42) in that paper, essentially because the SRS reactive cross sections are of hard-sphere type and the integral over $\mathbb{S}_{+}^{2}$ is explicitly evaluated in [67), whereas reactive cross sections in paper [5] are of Maxwell molecules type and the integral over $\mathbb{S}_{+}^{2}$ is not explicitly evaluated. Moreover, our exponent $1 / 2$, instead of $3 / 2$ as in paper [5], is a consequence of the fact that the exponent of the term $\left(\mu_{i j} / \mu_{k l}\right)$ in our reactive collision operator $J_{i}^{R}$ is 2 , see $[30]$, while in [5] it is 3 .

\subsection{Momentum balance equation}

The momentum balance equations for the species in the reactive mixture can formally be derived from the scaled equations (53), after multiplying by the molecular velocity $\mathbf{v}_{i}$ and then integrating over the velocity space. Also, the conservation laws and some of the fundamental properties stated in Section 3 will be used to compute explicitly the production terms appearing in the balance equations.

However, some words are needed before presenting the next lemma. As it is well known, the computation of the integral contributions appearing in the momentum balance equations is rather technical and extremely intricate, and the final explicit expressions of these contributions are quite huge. On the other hand, as it will become clear from the balance equations derived in the lemma (see 773 , below), only the $O(1)$ terms in $\alpha$ will be retained in the equations, that is only those terms associated to the elastic scattering will influence the final formulation of the balance equations. Accordingly, we include in the next lemma only the explicit expressions of the $O(1)$ terms and present in Appendix 6.2 the explicit expression of the $O\left(\alpha^{2}\right)$ terms. 
Lemma 4.3 The momentum balance equation for each of the species in the reactive mixture is given by

$$
\alpha^{2} \frac{\partial}{\partial t}\left(c_{i}^{\alpha} \mathbf{u}_{i}^{\alpha}\right)+\frac{k_{B} T}{m_{i}} \frac{\partial c_{i}^{\alpha}}{\partial \mathbf{x}}+\alpha^{2} \frac{\partial}{\partial \mathbf{x}}\left(c_{i}^{\alpha} \mathbf{u}_{i}^{\alpha} \otimes \mathbf{u}_{i}^{\alpha}\right)=\mathcal{O}_{i}-\mathcal{P}_{i}+\mathcal{Q}_{i},
$$

where $\mathcal{O}_{i}$ is an $O(1)$ production term associated to the elastic scattering, and is given by

$$
\mathcal{O}_{i}=\frac{1}{\alpha} \int_{\mathbb{R}^{3}} \mathbf{v}_{i} J_{i}^{b \alpha E} d \mathbf{v}_{i}=\frac{32}{9} \sum_{\substack{s=1 \\ s \neq i}}^{4} \sigma_{i s}^{2} \frac{m_{s}}{m_{i}+m_{s}}\left(\frac{2 \pi k_{B} T}{\mu_{i s}}\right)^{\frac{1}{2}} c_{i}^{\alpha} c_{s}^{\alpha}\left(\mathbf{u}_{s}^{\alpha}-\mathbf{u}_{i}^{\alpha}\right),
$$

and $\mathcal{P}_{i}, \mathcal{Q}_{i}$ are $O\left(\alpha^{2}\right)$ production terms associated to the chemical process, and are respectively given by

$$
\mathcal{P}_{i}=\alpha \int_{\mathbb{R}^{3}} \mathbf{v}_{i} J_{i j}^{b \alpha * E} d \mathbf{v}_{i}, \quad \mathcal{Q}_{i}=\alpha \int_{\mathbb{R}^{3}} \mathbf{v}_{i} J_{i}^{\alpha R} d \mathbf{v}_{i}
$$

whose explicit expressions are given in Appendix 6.2, see (91) and 104.

Proof. First, we multiply both sides of the scaled SRS equations given in 53 by $\mathbf{v}_{i}$ and integrate with respect $\mathbf{v}_{i} \in \mathbb{R}^{3}$, to obtain

$$
\begin{aligned}
\alpha \frac{\partial}{\partial t}\left(\int_{\mathbb{R}^{3}} \mathbf{v}_{i} f_{i}^{\alpha} d \mathbf{v}_{i}\right)+\frac{\partial}{\partial \mathbf{x}}\left(\int_{\mathbb{R}^{3}} \mathbf{v}_{i} \otimes \mathbf{v}_{i} f_{i}^{\alpha} d \mathbf{v}_{i}\right) & =\frac{1}{\alpha} \int_{\mathbb{R}^{3}} \mathbf{v}_{i} J_{i}^{m \alpha E} d \mathbf{v}_{i} \\
& +\underbrace{\frac{1}{\alpha} \int_{\mathbb{R}^{3}} \mathbf{v}_{i} J_{i}^{b \alpha E} d \mathbf{v}_{i}}_{O_{i}}-\underbrace{\alpha \int_{\mathbb{R}^{3}} \mathbf{v}_{i} J_{i j}^{b * \alpha E} d \mathbf{v}_{i}}_{\mathcal{P}_{i}}+\underbrace{\alpha \int_{\mathbb{R}^{3}} \mathbf{v}_{i} J_{i}^{\alpha R} d \mathbf{v}_{i}}_{\mathcal{Q}_{i}} .
\end{aligned}
$$

Let us concentrate first on the left-hand-side terms in 76 .

(i) From the second expression in (56), it immediately follows that

$$
\alpha \frac{\partial}{\partial t}\left(\int_{\mathbb{R}^{3}} \mathbf{v}_{i} f_{i}^{\alpha} d \mathbf{v}_{i}\right)=\alpha^{2} \frac{\partial}{\partial t}\left(c_{i}^{\alpha} \mathbf{u}_{i}^{\alpha}\right) .
$$

(ii) For what concerns the second term on the left-hand side of (76), we transform from $\mathbf{v}_{i}$ to the peculiar velocity $\boldsymbol{\xi}_{i}=\mathbf{v}_{i}-\alpha \mathbf{u}_{i}^{\alpha}$ and then use the fact that the Jacobian of the transformation is equal to 1 to obtain

$$
\begin{aligned}
\frac{\partial}{\partial \mathbf{x}}\left(\int_{\mathbb{R}^{3}} \mathbf{v}_{i} \otimes \mathbf{v}_{i} f_{i}^{\alpha} d \mathbf{v}_{i}\right) & =\frac{\partial}{\partial \mathbf{x}}\left(\int_{\mathbb{R}^{3}} f_{i}^{\alpha} \boldsymbol{\xi}_{i} \otimes \boldsymbol{\xi}_{i} d \boldsymbol{\xi}_{i}\right)+2 \alpha \frac{\partial}{\partial \mathbf{x}}\left(\mathbf{u}_{i}^{\alpha} \otimes \int_{\mathbb{R}^{3}} f_{i}^{\alpha} \boldsymbol{\xi}_{i} d \boldsymbol{\xi}_{i}\right) \\
& +\alpha^{2} \frac{\partial}{\partial \mathbf{x}}\left(\mathbf{u}_{i}^{\alpha} \otimes \mathbf{u}_{i}^{\alpha} \int_{\mathbb{R}^{3}} f_{i}^{\alpha} d \boldsymbol{\xi}_{i}\right) .
\end{aligned}
$$

Inserting 55, expressed in terms of the peculiar velocity $\boldsymbol{\xi}_{i}$, into 78 and evaluating the integrals, we easily see that the second term on the right hand side vanishes. Consequently, (78) reduces to

$$
\frac{\partial}{\partial \mathbf{x}}\left(\int_{\mathbb{R}^{3}} \mathbf{v}_{i} \otimes \mathbf{v}_{i} f_{i}^{\alpha} d \mathbf{v}_{i}\right)=\frac{k_{B} T}{m_{i}} \frac{\partial c_{i}^{\alpha}}{\partial \mathbf{x}}+\alpha^{2} \frac{\partial}{\partial \mathbf{x}}\left(c_{i}^{\alpha} \mathbf{u}_{i}^{\alpha} \otimes \mathbf{u}_{i}^{\alpha}\right)
$$

Now let us deal with the terms on the right hand side of 76 .

(iii) The first term vanishes by virtue of the second assertion in Corollary 3.11 about the mono-species elastic operator, see 41. 
(iv) To derive an explicit expression for the production term $\mathcal{O}_{i}$, we use the considered approximation of $f_{i}^{\alpha}$ in the form (57) or (64). Taking into account Lemma 3.8 with $\varphi\left(\mathbf{v}_{i}^{\prime}\right)=\mathbf{v}_{i}^{\prime}$ and $\varphi\left(\mathbf{v}_{i}\right)=\mathbf{v}_{i}$, we obtain

$$
\mathcal{O}_{i}=\frac{1}{\alpha} \sum_{\substack{s=1 \\ s \neq i}}^{4} \sigma_{i s}^{2} \int_{\mathbb{R}^{3}} \int_{\mathbb{R}^{3}} \int_{\mathbb{S}_{+}^{2}}\left(\mathbf{v}_{i}^{\prime}-\mathbf{v}_{i}\right) f_{i}^{\alpha} f_{s}^{\alpha}\left\langle\epsilon, \mathbf{v}_{i}-\mathbf{v}_{s}\right\rangle d \epsilon d \mathbf{v}_{s} d \mathbf{v}_{i} .
$$

Using the first expression in (14) for $\mathbf{v}_{i}^{\prime}$, evaluating the integral over the sphere $\mathbb{S}_{+}^{2}$ by transforming to spherical coordinates as described in Remark 3.1. and transforming the remaining sixfold integral in $\mathbf{v}_{i}$ and $\mathbf{v}_{s}$ to the relative velocity $\mathbf{V}^{*}=\mathbf{v}_{s}-\mathbf{v}_{i}$ and centre of mass velocity $\mathbf{X}^{*}=\left(m_{i} \mathbf{v}_{i}+m_{s} \mathbf{v}_{s}\right) / M_{i s}$, with $M_{i s}=m_{i}+m_{s}$, as defined before, we obtain

$$
\begin{aligned}
\mathcal{O}_{i}=\frac{4 \pi}{3 \alpha} \sum_{\substack{s=1 \\
s \neq i}}^{4} \sigma_{i s}^{2} \frac{m_{s}}{M_{i s}} c_{i}^{\alpha} c_{s}^{\alpha} \frac{\left(m_{i} m_{s}\right)^{\frac{3}{2}}}{\left(2 \pi k_{B} T\right)^{3}} \\
\times\left\{\left(\int_{\mathbb{R}^{3}} \exp \left(-\frac{M_{i s} X^{* 2}}{2 k_{B} T}\right) d \mathbf{X}^{*}\right)\left(\int_{\mathbb{R}^{3}} V^{*} \exp \left(-\frac{\mu_{i s} V^{* 2}}{2 k_{B} T}\right) \mathbf{V}^{*} d \mathbf{V}^{*}\right)\right. \\
+\left(\int_{\mathbb{R}^{3}} \exp \left(-\frac{M_{i s} X^{* 2}}{2 k_{B} T}\right) \mathbf{X}^{*} d \mathbf{X}^{*}\right)\left(\mathbf{a}_{s}+\mathbf{a}_{i}\right) \cdot\left(\int_{\mathbb{R}^{3}} V^{*} \exp \left(-\frac{\mu_{i s} V^{* 2}}{2 k_{B} T}\right) \mathbf{V}^{*} d \mathbf{V}^{*}\right) \\
+\frac{m_{i}}{M_{i s}}\left(\int_{\mathbb{R}^{3}} \exp \left(-\frac{M_{i s} X^{* 2}}{2 k_{B} T}\right) d \mathbf{X}^{*}\right)\left(\int_{\mathbb{R}^{3}}\left(\mathbf{a}_{s} \cdot \mathbf{V}^{*}\right) \mathbf{V}^{*} \exp \left(-\frac{\mu_{i s} V^{* 2}}{2 k_{B} T}\right) V^{*} d \mathbf{V}^{*}\right) \\
\\
\left.\quad-\frac{m_{s}}{M_{i s}}\left(\int_{\mathbb{R}^{3}} \exp \left(-\frac{M_{i s} X^{* 2}}{2 k_{B} T}\right) d \mathbf{X}^{*}\right)\left(\int_{\mathbb{R}^{3}}\left(\mathbf{a}_{i} \cdot \mathbf{V}^{*}\right) \mathbf{V}^{*} \exp \left(-\frac{\mu_{i s} V^{* 2}}{2 k_{B} T}\right) V^{*} d \mathbf{V}^{*}\right)\right\},
\end{aligned}
$$

where we have neglected the quadratic term in the coefficients a and introduced the notation $V^{*}=\left\|\mathbf{V}^{*}\right\|$, $X^{*}=\left\|\mathbf{X}^{*}\right\|$. Also, $\mathbf{a}_{i}=\frac{\alpha m_{i} \mathbf{u}_{i}^{\alpha}}{k_{B} T}, \mathbf{a}_{\mathbf{s}}=\frac{\alpha m_{i} \mathbf{u}_{s}^{\alpha}}{k_{B} T}$ as defined in (64).

Considering the first integral in 80 , writing $\mathbf{V}^{*}=V^{*} \overrightarrow{\mathbf{v}}$ with $\overrightarrow{\mathbf{v}}$ a unit vector, and transforming to spherical coordinates, we conclude that the integral vanishes, since

$$
\begin{aligned}
\int_{\mathbb{R}^{3}} V^{*} \exp \left(-\frac{\mu_{i s} V^{* 2}}{2 k_{B} T}\right) \mathbf{V}^{*} d \mathbf{V}^{*} & =\int_{0}^{\infty} V^{* 4} \exp \left(-\frac{\mu_{i s} V^{* 2}}{2 k_{B} T}\right) d V^{*}\left(\int_{0}^{\pi} \sin ^{2} \theta d \theta \int_{0}^{2 \pi} \cos \phi d \phi \hat{\mathbf{x}}\right. \\
& \left.+\int_{0}^{\pi} \sin ^{2} \theta d \theta \int_{0}^{2 \pi} \sin \phi d \phi \hat{\mathbf{y}}+\int_{0}^{\pi} \sin \theta \cos \theta d \theta \int_{0}^{2 \pi} d \phi \hat{\mathbf{z}}\right)=0
\end{aligned}
$$

where $\hat{\mathbf{x}}, \hat{\mathbf{y}}, \hat{\mathbf{z}}$ are the Cartesian unit vectors in $\mathbb{R}^{3}$. This implies that the first two addends within the braces in 80 vanish. Concerning now the integrals in the third and last terms on the right hand side of the same equation, we have that the integrals in $\mathbf{X}^{*}$ are similar to the one in $(69)$. Moreover, by setting $\left(\mathbf{a}_{s} \cdot \mathbf{V}^{*}\right) \mathbf{V}^{*}=a_{s} \cos \theta V^{* 2} \overrightarrow{\mathbf{v}}$, with $a_{s}=\left\|\mathbf{a}_{s}\right\|$, in the integral with respect to $\mathbf{V}^{*}$ appearing in the third term, and then transforming to spherical coordinates, we obtain

$$
\begin{array}{r}
\int_{\mathbb{R}^{3}} a_{s} \cos \theta V^{* 2} \overrightarrow{\mathbf{v}} \exp \left(-\frac{\mu_{i s} V^{* 2}}{2 k_{B} T}\right) V^{*} d \mathbf{V}^{*}=a_{s} \int_{0}^{\infty} V^{* 5} \exp \left(-\frac{\mu_{i j} V^{* 2}}{2 k_{B} T}\right) d V^{*}\left(\hat{\mathbf{x}} \int_{0}^{\pi} \sin ^{2} \theta \cos \theta d \theta\right. \\
\underbrace{\int_{0}^{2 \pi} \cos \phi d \phi}_{0}+\hat{\mathbf{y}} \int_{0}^{\pi} \sin ^{2} \theta \cos \theta d \theta \underbrace{\int_{0}^{2 \pi} \sin \phi d \phi}_{0}+\hat{\mathbf{z}} \underbrace{\int_{0}^{\pi} \sin \theta \cos ^{2} \theta d \theta}_{\frac{2}{3}} \underbrace{\int_{0}^{2 \pi} d \phi}_{2 \pi}) .
\end{array}
$$

Performing the integration with respect to $V^{*}$, we obtain

$$
\int_{\mathbb{R}^{3}}\left(\mathbf{a}_{s} \cdot \mathbf{V}^{*}\right) \mathbf{V}^{*} \exp \left(-\frac{\mu_{i s} V^{* 2}}{2 k_{B} T}\right) V^{*} d \mathbf{V}^{*}=\frac{4 \pi}{3}\left(\frac{2 k_{B} T}{\mu_{i s}}\right)^{3} \mathbf{a}_{s} .
$$


Similarly, with respect to the last term on the right hand side of 80 , we obtain

$$
\int_{\mathbb{R}^{3}}\left(\mathbf{a}_{i} \cdot \mathbf{V}^{*}\right) \mathbf{V}^{*} \exp \left(-\frac{\mu_{i s} V^{* 2}}{2 k_{B} T}\right) V^{*} d \mathbf{V}^{*}=\frac{4 \pi}{3}\left(\frac{2 k_{B} T}{\mu_{i s}}\right)^{3} \mathbf{a}_{i}
$$

Substituting the above results into 80 and performing a little algebra we derive the final expression (74). This ends the proof of Lemma 4.3. The computation of the $O\left(\alpha^{2}\right)$ terms $\mathcal{P}_{i}$ and $\mathcal{Q}_{i}$ is omitted here, see Appendix 6.2 ,

Remark 4.4 (a) The diffusion coefficients in our limiting equations can be computed explicitly from expressions (74). This will be done in the next subsection, see expression 866).

(b) In paper [25], for another kinetic model, the authors considered a different input function (see Eq. (32) of [25]) and obtained a production term (see (34) in that paper) similar to the one in our expression (74). However, the coefficient 32/9 in our case, instead of 8/3 in paper [25], results from the fact that the models are different and also from the definition of the elastic cross sections, namely we use $\sigma_{i s}^{2}=\left(d_{i}+d_{s}\right)^{2} / 4$ and paper [25] uses $\sigma_{\beta \alpha}=\left(d_{\alpha}+d_{\beta}\right)^{2} / 16$.

\subsection{Macroscopic equations and formal asymptotics}

In this subsection we state the main result of this paper. In particular, using Lemmas 4.1 and 4.3, we formally derive the reaction diffusion system of MS type as the hydrodynamic asymptotic limit of the scaled SRS kinetic system (53). The connection between the two systems is based on the fact that the scaled Maxwellians (55) solve the kinetic equations if the macroscopic parameters $c_{i}^{\alpha}$ and $\mathbf{u}_{i}^{\alpha}$ characterizing such Maxwellians solve the approximate equations $(60)$ and $(73)$. The conclusion is obtained in the limit as $\alpha \rightarrow 0$ by assuming that the functions $c_{i}^{\alpha}, \mathbf{u}_{i}^{\alpha}$ converge pointwise to $c_{i}, \mathbf{u}_{i}$, for $t \geq 0$ and $\mathbf{x} \in \Omega$.

Theorem 4.5 (i) The Maxwellians defined in (55) are solutions of the initial boundary value problem for the scaled SRS kinetic equations (53) with initial conditions (54) if, for $i=1,2,3,4$, functions $c_{i}^{\alpha}$ and $\mathbf{u}_{i}^{\alpha}$ solve the approximate system

$$
\left\{\begin{array}{l}
\frac{\partial c_{i}^{\alpha}}{\partial t}+\frac{\partial}{\partial \mathbf{x}}\left(c_{i}^{\alpha} \mathbf{u}_{i}^{\alpha}\right)=\mathcal{J}_{i}, \\
\alpha^{2} \frac{\partial}{\partial t}\left(c_{i}^{\alpha} \mathbf{u}_{i}^{\alpha}\right)+\frac{k_{B} T}{m_{i}} \frac{\partial c_{i}^{\alpha}}{\partial \mathbf{x}}+\alpha^{2} \frac{\partial}{\partial \mathbf{x}}\left(c_{i}^{\alpha} \mathbf{u}_{i}^{\alpha} \otimes \mathbf{u}_{i}^{\alpha}\right)=\mathcal{O}_{i}-\mathcal{P}_{i}+\mathcal{Q}_{i},
\end{array}\right.
$$

where the reaction rate $\mathcal{J}_{i}$ and the production terms $\mathcal{O}_{i}, \mathcal{P}_{i}$ and $\mathcal{Q}_{i}$, defined respectively by 61, ,74) and (75), are computed using the approximations (57) to the Maxwellians (55).

(ii) Moreover, in the limit as $\alpha \rightarrow 0$, the system 83 reduces to

$$
\left\{\begin{aligned}
& \frac{\partial c_{i}}{\partial t}+\frac{\partial \mathbf{J}_{i}}{\partial \mathbf{x}}=\sigma_{i j}^{2}\left(\frac{2 \pi \mu_{i j}}{k_{B} T}\right)^{\frac{1}{2}} {\left[\left(\frac{\mu_{i j}}{\mu_{k l}}\right)^{\frac{1}{2}} c_{k} c_{l} \exp \left(\frac{Q_{R}}{k_{B} T}\right)-c_{i} c_{j}\right] } \\
& \times\left[\frac{2 k_{B} T}{\mu_{i j}} \Gamma\left(2, z_{i}^{*}\right)-\left(\frac{\Xi_{i j}}{c_{0}}\right)^{2} \Gamma\left(1, z_{i}^{*}\right)\right], \\
& \frac{\partial c_{i}}{\partial \mathbf{x}}=\frac{32}{9} \sum_{\substack{s=1 \\
s \neq i}}^{4} \sigma_{i s}^{2}\left(\frac{2 \pi \mu_{i j}}{k_{B} T}\right)^{\frac{1}{2}}\left(c_{i} \mathbf{J}_{s}-c_{s} \mathbf{J}_{i}\right),
\end{aligned}\right.
$$

whose unknowns are the concentrations $c_{i}$ and the diffusive fluxes $\mathbf{J}_{i}$. 
Proof. The statement in item (i) follows from Lemmas 4.1 and 4.3

Concerning item (ii), we can write $c_{i}^{\alpha} \mathbf{u}_{i}^{\alpha}=\mathbf{J}_{i}^{\alpha}+c_{i}^{\alpha} \mathbf{u}^{\alpha}$, where $\mathbf{u}^{\alpha}$ represents the average velocity of the mixture relative to the scaled distributions $f_{i}^{\alpha}$, see expression (50b). Letting $\alpha \rightarrow 0$ in (83), assuming that the limits

$$
c_{i}=\lim _{\alpha \rightarrow 0} c_{i}^{\alpha}, \quad \mathbf{J}_{i}=\lim _{\alpha \rightarrow 0} \mathbf{J}_{i}^{\alpha}, \quad \mathbf{u}=\lim _{\alpha \rightarrow \mathbf{0}} \mathbf{u}^{\alpha},
$$

exist pointwise for any $t>0$ and $\mathbf{x} \in \Omega$, and neglecting the convective term, that is $\frac{\partial}{\partial \mathbf{x}}\left(c_{i} \mathbf{u}\right)=\mathbf{0}$, we obtain the desired system (84).

Observe that summing the first and second equations of (84) over all species, we obtain that $c$ is uniform in space and constant in time. Therefore, the second equation of system 84 can be rewritten as

$$
\frac{\partial c_{i}}{\partial \mathbf{x}}=\frac{1}{c} \sum_{\substack{s=1 \\ s \neq i}}^{4} \frac{c_{i} \mathbf{J}_{s}-c_{s} \mathbf{J}_{i}}{D_{i s}},
$$

where

$$
D_{i s}=\frac{9}{32}\left(\frac{k_{B} T}{2 \pi \mu_{i s}}\right)^{\frac{1}{2}} \frac{1}{c \sigma_{i s}^{2}}, \quad i \neq s
$$

are the diffusion coefficients.

Consequently, for $i=1,2,3,4$, putting together the constraint law (6), the boundary conditions (9) for the diffusive fluxes $\mathbf{J}_{i}$, and 84 with the gradient term $\frac{\partial c_{i}}{\partial \mathbf{x}}$ expressed as given in 85, we obtain

$$
\left\{\begin{array}{l}
\frac{\partial c_{i}}{\partial t}+\frac{\partial \mathbf{J}_{i}}{\partial \mathbf{x}}=\mathcal{J}_{i}, \quad \mathbf{x} \in \Omega, \quad t>0, \\
\frac{\partial c_{i}}{\partial \mathbf{x}}=-\frac{1}{c} \sum_{\substack{s=1 \\
s \neq i}}^{4} \frac{c_{s} \mathbf{J}_{i}-c_{i} \mathbf{J}_{s}}{D_{i s}}, \quad \mathbf{x} \in \Omega, \quad t>0, \\
\sum_{i=1}^{4} m_{i} \mathbf{J}_{i}=0, \quad \mathbf{x} \in \Omega, \quad t>0, \\
\nu \cdot \mathbf{J}_{i}=0, \quad \mathbf{x} \in \partial \Omega, \quad t>0 .
\end{array}\right.
$$

System (87) constitutes a boundary value problem which we refer to as the reaction diffusion system of MaxwellStefan type.

Remark 4.6 It is important to underline that expansion (57) was used in this paper for the explicit computation of the integrals appearing on the right hand side of the concentration and momentum balance equations (see equations (60) and (73), respectively).

Furthermore, we note that a system of Maxwell-Stefan type with the same general structure of the one in (84) can be derived from the SRS kinetic model if we do not use expansion (57).

\section{Conclusion}

In this paper, we formally derive a reaction diffusion system of Mawell-Stefan type as the hydrodynamic limit of the simple reacting spheres kinetic model for a quaternary mixture of monatomic ideal gases undergoing a reversible chemical reaction of bimolecular type. By considering a scaling in which elastic collisions play a dominant role in the evolution of the species while chemical reactions are slow, and using a first order correction to the Maxwellian distribution in the species rest frame, the diffusion coefficients and the chemical production 
rates appearing in the species equations for the concentration and momentum have been explicitly computed from the collisional dynamics of the kinetic model.

An important aspect in our work is that we have used the same correction to the Maxwellian distribution for the computation of both elastic and reactive production terms, leading to a more consistent macroscopic picture (see Remark 4.6). Moreover, our correction to the Maxwellian distribution coincides with the one used by Stefan in his celebrated work from 1872 to derive the diffusion coefficients in a mixture of gases.

In the quoted literature about the hydrodynamic limits of kinetic models for reactive mixtures, the derivation of the MS equations had not yet been considered. Our work provides the first result in this direction and, in our opinion, complements the work developed in [11, 12, 13, 22] in the context of non-reactive mixtures.

Still in connection with the work developed in [11, 12, 13, 22] for non-reactive mixtures, we would like to emphasize here that when we turn off the chemical reaction in our model (i.e if the mixture is made up of four non-reactive species), our limiting system (87) reduces to the Maxwell-Stefan system for hard sphere molecules, which is similar to the one obtained in 12 for Maxwellian molecules.

The fact that in our analysis the mixture can react chemically allows to consider many interesting problems concerning the derivation of the MS equations from the kinetic model. Among the most interesting problems, we quote the following.

The first problem is the introduction of chemical potentials as the main agent in the definition of the driving forces and the study of the passage to the hydrodynamic limit by removing the isothermal assumption. This will certainly lead to a very rich setting from the physical but also from the mathematical point of view.

The second problem concerns the possibility of studying different time scales associated to the chemical reaction, in particular different chemical regimes, and obtaining the influence of chemical reaction in the limiting MS equations.

In fact, the scaling of slow chemical reaction studied here is the sole reason why the limit equations for the momentum of the species do not include any contributions coming from the reactive collision terms. This can be seen from Lemma 4.3 , in particular, from the second equation in (83), where clearly the chemical contributions are present, but they are of order of $\alpha^{2}$, due to the scaling, and hence go to zero in the limit as $\alpha \rightarrow 0$.

Additionally, when studying different time scales associated to the chemical reaction, especially those chemical regimes in which reactive collisions are treated on a par with elastic ones, our SRS model results to be appropriate, because of the inclusion of the correction term in the elastic operator. This correction term, as mentioned before, singles out those pre-collisional states that are energetic enough to result in a chemical reaction, therefore preventing double counting of these collisions in the elastic and reactive operators. In this sense, the correction term leads to non anomalous results.

Finally, our next line of action will include an extension of the present analysis to kinetic models for reactive mixtures with polyatomic structure, in view of describing more meaningful chemical processes.

The problems just described above will be addressed in future works.

Acknowledgments. B. A. and A. J. S. thank Centro de Matemática da Universidade do Minho, Portugal, and the FCT/Portugal Project UID/MAT/00013/2013. B. A. thanks the FCT/Portugal for the support through the PhD grant PD/BD/128188/2016. P. G. thanks FCT/Portugal for the support through the project UID/MAT/04459/2013 and the French Ministry of Education through the grant ANR (EDNHS). The authors thank the Program Pessoa of Cooperation between Portugal and France with reference 406/4/4/2017/S. 


\section{References}

[1] B.V. Alexeev, A. Chikhaoui and I.T. Grushin, Application of the generalized Chapman-Enskog method to the transport-coefficient calculation in a reacting gas mixture, Phys. Rev. E, 49, 2809-2825 1994.

[2] C. Bardos, F. Golse, C.D. Levermore, Sur les limites asymptotiques de la théorie cinétique conduisant à la dynamique des fluides incompressibles, C. R Math. Acad. Sci., 309, 727-732, 1989.

[3] C. Bardos, F. Golse, C.D. Levermore, Fluid dynamic limits of kinetic equations. I. Formal derivations, J. Stat. Phys., 63, 323-344, 1991.

[4] C. Bardos, F. Golse, C. D. Levermore, Fluid dynamic limits of kinetic equations. II. Convergence proofs for the Boltzmann equation, Commun. Pure Appl. Math, 46, 667-753, 1993.

[5] M. Bisi, L. Desvillettes, From reactive Boltzmann equations to reaction-diffusion systems, J. Stat. Phys., 124, 881-912, 2006.

[6] M. Bisi, L. Desvillettes, Some remarks about the scaling of systems of reactive Boltzmann equations, Kinetic and Related Models, 1, 515-520, 2008.

[7] M. Bisi, G. Spiga, Diatomic gas diffusing in a background medium: kinetic approach and reaction-diffusion equations, Commun. Math. Sci., 4, 779-798, 2006.

[8] D. Bothe, On the Maxwell-Stefan approach to multicomponent diffusion, Progress in Nonliniear Differential Equation and Their Applications, 80, 81-93, 2011.

[9] D. Bothe, W. Dreyer, Continuum thermodynamics of chemically reacting fluid mixtures, Acta Mech., 226, $1757-1805,2015$.

[10] D. Bothe, A. Fischer, M. Pierre, G. Rolland, Global wellposedness for a class of reaction-advectionanisotropic diffusion systems, J. Evol. Equ., 17, 101-130, 2017.

[11] L. Boudin, B. Grec, F. Salvarani, A mathematical and numerical analysis of the Maxwell-Stefan diffusion equations, Discrete Contin. Dyn. Syst., Ser. B, 17, 1427-1440, 2012.

[12] L. Boudin, B. Grec, F. Salvarani, The Maxwell-Stefan diffusion limit for a kinetic model of mixtures, Acta Appl. Math., 136, 79-90, 2015.

[13] L. Boudin, B. Grec, V. Pavan, The Maxwell-Stefan diffusion limit for a kinetic model of mixtures with general cross sections, Nonlinear Analysis, 159, 40-61, 2017.

[14] F. Carvalho, Mathematical methods for the Boltzmann equation in the context of chemically reactive gases. Doctoral thesis. http://hdl.handle.net/1822/24430 (2013). Repositorium of the University of Minho.

[15] J. S. Dahler, L. Qin, Nonequilibrium statistical mechanics of chemically reactive fluids, J. Chem. Physics, 118, 8396-8404, 2003

[16] D.D. Do, Adsorption analysis: equilibria and kinetics, Imperial College Press, London, 1998.

[17] V. Giovangigli, Multicomponent Flow Modeling, Birkhäuser Boston, 1999.

[18] F. Golse, The Boltzmann equation and its hydrodynamic limits, Handbook of differential equations, evolutionary equations, Volume II, Eds. C.M. Dafermos and E. Feireisl, Elsevier, 309, 159-301, 2005.

[19] F. Golse, Fluid dynamic limits of the kinetic theory of gases, From Particle Systems to Partial Differential Equations I, Springer Proceedings in Mathematics and Statistics, 75, 1-91, 2014.

[20] M. Groppi, J. Polewczak, On two kinetic models for chemical reactions: comparisons and existence results, J. Stat. Physics, 117, 211-241, 2004.

[21] M. Herberg, M. Meyries, J. Prüss, M. Wilke, Reaction-diffusion systems of Maxwell-Stefan type with reversible mass-action kinetics, Nonlinear Analysis, 159, 264-284, 2017. 
[22] H. Hutridurga, F. Salvarani, On the Maxwell-Stefan diffusion limit for a mixture of monatomic gases, Math. Meth. Appl. Sci., 40, 803-813, 2017.

[23] A. Jüngel, I. V. Stelzer, Existence analysis of Maxwell-Stefan systems for multicomponent mixtures, J. Math. Anal., 45, 2421-2440, 2013.

[24] G. M. Kremer, An introduction to the Boltzmann equation and transport processes in gases, Springer, Berlin, 2010.

[25] G.M. Kremer, M.P. Bianchi, A.J. Soares, Analysis of the trend to equilibrium of a chemically reacting system, J. Phys. A: Math. Theor., 40, 2553-2571, 2007.

[26] R. Krishna, R. Taylor, Multicomponent mass transfer theory and applications, In N. Cheremisinoff (ed.), Handbook for heat and mass transfer, 2, Gulf Houston, 1986.

[27] R. Krishna, J. Wesselingh, The Maxwell-Stefan approach to mass transfer, Chem. Engin. Sci., 52, 861-911, 1997.

[28] M .T. Marron, Simple collision theory of reactive hard spheres, J. Chem. Phys., 52, 4060-4061, 1970.

[29] J. Polewczak, The kinetic theory of simple reacting spheres I. Global existence result in dilute gas case, J. Stat. Phys., 100, 327-362, 2000.

[30] J. Polewczak, A. J. Soares, On modified simple reacting spheres kinetic model for chemically reactive gases, Kinetic and Related Models, 10, 513-539, 2017.

[31] R.D. Present, Kinetic theory of gases, New York, McGraw-Hill, 1958.

[32] L. Qin, J. S. Dahler, The kinetic theory of a simple reacting fluid: Scattering functions and relaxation processes, J. Chem. Phys., 103, 72-750, 1995.

[33] R. Spigler, D.H. Zanette, Reaction-diffusion models from the Fokker-Planck formulation of chemical processes, IMA J. Appl. Math., 49, 217-229, 1992.

[34] R. Spigler, D.H. Zanette, Asymptotic analysis and reaction-diffusion approximation for BGK kinetic models of chemical processes in multispecies gas mixtures, J. Appl. Math. Phys. (ZAMP), 44, 812-827., 1993.

[35] R. Taylor, R. Krishna, Multicomponent mass transfer, Wiley, New York, 1993.

[36] Van den Broeke, R. Krishna, Experimental verification of the Maxwell-Stefan theory for micropore diffusion, Chem. Engin. Sci., 50, 2507-2522, 1995.

[37] C. Villani, A review of mathematical topics in collisional kinetic theory, in the Handbook of Mathematical Fluid Dynamics, Volume I, Chapter 2, Eds. S. Friedlander and D. Serre, 71-305, Elsevier, 2002.

[38] N. Xystris, J. S. Dahler, Kinetic theory of simple reacting spheres, J. Chem. Physics, 68, 387-401, 1978.

[39] J. Wesselingh, R. Krishna, Mass transfer in multicomponent mixtures, Delft University Press, 2006.

[40] D.H. Zanette, Linear and nonlinear diffusion and reaction-diffusion equations from discrete-velocity kinetic models, J. Phys. A: Math. Gen., 26, 5339-5349, 1993.

[41] S. S. Zumdahl, S. A. Zumdahl, Chemistry: An atoms first approach Brooks/cole, CENGAGE Learning, Belmont, 2012.

[42] H. Hutridurga, F. Salvarani, Maxwell-Stefan diffusion asymptotics for gas mixtures in non-isothermal setting, Nonlinear Analysis, 159, 285-297, 2017.

[43] E. Zatorska, On the steady flow of a multicomponent, compressible, chemically reacting gas, Nonlinearity, 24, 3267-3278, 2011.

[44] E. Zatorska, P. B. Mucha, Multicomponent mixture model: the issue of existence via time discretization, Commun. Math. Sci., 13, 1975-2003, 2015. 


\section{Appendices}

In this section, we provide some details about the computation of the integrals given in Section 4 that were not evaluated there.

\subsection{Appendix A}

In this appendix, we prove that $\mathcal{E}$ and $\mathcal{F}$, given in 65 of Subsection 4.2 are null. We start with $\mathcal{E}$ and write it as the sum of the next two terms

$$
\begin{aligned}
\mathcal{E}_{1} & =\sigma_{i j}^{2}\left(\frac{\mu_{i j}}{\mu_{k l}}\right)^{2} \mathbf{a}_{k} \cdot \int_{\mathbb{R}^{3}} \int_{\mathbb{R}^{3}} \int_{\mathbb{S}_{+}^{2}} \mathbf{v}_{k}^{\circ} M_{k}^{\alpha} M_{l}^{\alpha} \Theta\left(\left\langle\epsilon, c_{0} \mathbf{v}_{i}-c_{0} \mathbf{v}_{j}\right\rangle-\Xi_{i j}\right)\left\langle\epsilon, \mathbf{v}_{i}-\mathbf{v}_{j}\right\rangle d \epsilon d \mathbf{v}_{j} d \mathbf{v}_{i}, \\
\mathcal{E}_{2} & =\sigma_{i j}^{2}\left(\frac{\mu_{i j}}{\mu_{k l}}\right)^{2} \mathbf{a}_{l} \cdot \int_{\mathbb{R}^{3}} \int_{\mathbb{R}^{3}} \int_{\mathbb{S}_{+}^{2}} \mathbf{v}_{l}^{\circ} M_{k}^{\alpha} M_{l}^{\alpha} \Theta\left(\left\langle\epsilon, c_{0} \mathbf{v}_{i}-c_{0} \mathbf{v}_{j}\right\rangle-\Xi_{i j}\right)\left\langle\epsilon, \mathbf{v}_{i}-\mathbf{v}_{j}\right\rangle d \epsilon d \mathbf{v}_{j} d \mathbf{v}_{i} .
\end{aligned}
$$

From (58) we rewrite the integral appearing in $\mathcal{E}_{1}$ as

$$
\begin{aligned}
c_{k}^{\alpha} c_{l}^{\alpha} \frac{\left(m_{k} m_{l}\right)^{\frac{3}{2}}}{\left(2 \pi k_{B} T\right)^{3}} \int_{\mathbb{R}^{3}} \int_{\mathbb{R}^{3}} \int_{\mathbb{S}_{+}^{2}} \mathbf{v}_{k}^{\circ} \exp \left[-\frac{m_{k}\left(\mathbf{v}_{k}^{\circ}\right)^{2}+m_{l}\left(\mathbf{v}_{l}^{\circ}\right)^{2}}{2 k_{B} T}\right] & \\
& \times \Theta\left(\left\langle\epsilon, c_{0} \mathbf{v}_{i}-c_{0} \mathbf{v}_{j}\right\rangle-\Xi_{i j}\right)\left\langle\epsilon, \mathbf{v}_{i}-\mathbf{v}_{j}\right\rangle d \epsilon d \mathbf{v}_{j} d \mathbf{v}_{i} .
\end{aligned}
$$

Now we change variables from $\mathbf{v}_{i}, \mathbf{v}_{j}$ to $\mathbf{v}_{k}^{\circ}, \mathbf{v}_{l}^{\circ}$ and use the first expression of 26] given in Property 3.4 together with 25) to rewrite the previous term as

$$
\begin{aligned}
c_{k}^{\alpha} c_{l}^{\alpha} \frac{\left(m_{k} m_{l}\right)^{\frac{3}{2}}}{\left(2 \pi k_{B} T\right)^{3}}\left(\frac{\mu_{k l}}{\mu_{i j}}\right)^{2} \int_{\mathbb{R}^{3}} \int_{\mathbb{R}^{3}} \int_{\mathbb{S}_{+}^{2}} \mathbf{v}_{k}^{\circ} \exp \left[-\frac{m_{k}\left(\mathbf{v}_{k}^{\circ}\right)^{2}+m_{l}\left(\mathbf{v}_{l}^{\circ}\right)^{2}}{2 k_{B} T}\right] \\
\quad \times \Theta\left(\left\langle\epsilon, c_{0} \mathbf{v}_{k}^{\circ}-c_{0} \mathbf{v}_{l}^{\circ}\right\rangle-\Xi_{k l}\right)\left\langle\epsilon, \mathbf{v}_{k}^{\circ}-\mathbf{v}_{l}^{\circ}\right\rangle d \epsilon d \mathbf{v}_{k}^{\circ} d \mathbf{v}_{l}^{\circ} .
\end{aligned}
$$

Performing similar computations to those of (67), we conclude that the integral in 88 is equal to the difference of the next two integrals

$$
\begin{aligned}
& \mathcal{E}_{1}^{1}=\int_{\mathbb{R}^{3}} \int_{\mathbb{R}^{3}} \mathbf{v}_{k}^{\circ} \exp \left[-\frac{m_{k}\left(\mathbf{v}_{k}^{\circ}\right)^{2}+m_{l}\left(\mathbf{v}_{l}^{\circ}\right)^{2}}{2 k_{B} T}\right] V_{*} \Theta\left(V_{*}-\frac{\Xi_{k l}}{c_{0}}\right) d \mathbf{v}_{k}^{\circ} d \mathbf{v}_{l}^{\circ}, \\
& \mathcal{E}_{1}^{2}=\int_{\mathbb{R}^{3}} \int_{\mathbb{R}^{3}} \mathbf{v}_{k}^{\circ} \exp \left[-\frac{m_{k}\left(\mathbf{v}_{k}^{\circ}\right)^{2}+m_{l}\left(\mathbf{v}_{l}^{\circ}\right)^{2}}{2 k_{B} T}\right] V_{*} \Theta\left(V_{*}-\frac{\Xi_{k l}}{c_{0}}\right)\left(\frac{\Xi_{k l}}{c_{0} V_{*}}\right)^{2} d \mathbf{v}_{k}^{\circ} d \mathbf{v}_{l}^{\circ},
\end{aligned}
$$

where $V_{*}=\left\|\mathbf{v}_{k}^{\circ}-\mathbf{v}_{l}^{\circ}\right\|=\left\|\mathbf{v}_{l}^{\circ}-\mathbf{v}_{k}^{\circ}\right\|$. Now, we look first at $\mathcal{E}_{1}^{1}$. We transform the six fold integral in $\mathbf{v}_{k}^{\circ}$ and $\mathbf{v}_{l}^{\circ}$ to the centre of mass velocity $\mathbf{X}_{*}=\left(m_{k} \mathbf{v}_{k}^{\circ}+m_{l} \mathbf{v}_{l}^{\circ}\right) / M$ and relative velocity $\mathbf{V}_{*}=\mathbf{v}_{l}^{\circ}-\mathbf{v}_{k}^{\circ}$. Using the fact that the Jacobian of the transformation has absolute value 1, we obtain

$$
\begin{aligned}
\mathcal{E}_{1}^{1} & =\int_{\mathbb{R}^{3}} \mathbf{X}_{*} \exp \left(-\frac{M X_{*}^{2}}{2 k_{B} T}\right) d \mathbf{X}_{*} \int_{\mathbb{R}^{3}} \exp \left(-\frac{\mu_{k l} V_{*}^{2}}{2 k_{B} T}\right) V_{*} \Theta\left(V_{*}-\frac{\Xi_{k l}}{c_{0}}\right) d \mathbf{V}_{*} \\
& -\frac{m_{l}}{M} \int_{\mathbb{R}^{3}} \exp \left(-\frac{M X_{*}^{2}}{2 k_{B} T}\right) d \mathbf{X}_{*} \int_{\mathbb{R}^{3}} \mathbf{V}_{*} \exp \left(-\frac{\mu_{k l} V_{*}^{2}}{2 k_{B} T}\right) V_{*} \Theta\left(V_{*}-\frac{\Xi_{k l}}{c_{0}}\right) d \mathbf{V}_{*},
\end{aligned}
$$

where $X_{*}=\left\|\mathbf{X}_{*}\right\|$. Note that the integral with respect to $\mathbf{X}_{*}$ in the first term on the right hand side of $\mathcal{E}_{1}^{1}$ is null and so the first term above vanishes. Moreover, the integral with respect to $\mathbf{V}_{*}$ in the second term can be written in spherical coordinates and it is equal to

$$
\int_{V_{*} \geq \frac{\Xi_{k l}}{c_{0}}}^{\infty} \int_{0}^{\pi} \int_{0}^{2 \pi} V_{*}^{4} \overrightarrow{\mathbf{v}} \exp \left(-\frac{\mu_{k l} V_{*}^{2}}{2 k_{B} T}\right) \sin \theta d \phi d \theta d V_{*},
$$


where we used the fact that any vector can be written in terms of a unit vector, i.e. $\mathbf{V}_{*}=V_{*} \overrightarrow{\mathbf{v}}$, with $\overrightarrow{\mathbf{v}}=$ $\hat{\mathbf{x}} \sin \theta \cos \phi+\hat{\mathbf{y}} \sin \theta \sin \phi+\hat{\mathbf{z}} \cos \theta$, and $\hat{\mathbf{x}}, \hat{\mathbf{y}}, \hat{\mathbf{z}}$ the Cartesian unit vectors in $\mathbb{R}^{3}$. By simple computations, we get that the previous integral is equal to zero. This shows that $\mathcal{E}_{1}^{1}=0$. Now, repeating exactly the same strategy as above in order to show that $\mathcal{E}_{1}^{2}=0$, it is enough to compute the next two integrals

$$
\begin{aligned}
& \mathcal{E}_{1 A}^{2}=\int_{\mathbb{R}^{3}} \mathbf{X}_{*} \exp \left(-\frac{M X_{*}^{2}}{2 k_{B} T}\right) d \mathbf{X}_{*} \int_{\mathbb{R}^{3}} \exp \left(-\frac{\mu_{k l} V_{*}^{2}}{2 k_{B} T}\right) V_{*} \Theta\left(V_{*}-\frac{\Xi_{k l}}{c_{0}}\right)\left(\frac{\Xi_{k l}}{c_{0} V_{*}}\right)^{2} d \mathbf{V}_{*}, \\
& \mathcal{E}_{1 B}^{2}=\int_{\mathbb{R}^{3}} \mathbf{V}_{*} \exp \left(-\frac{\mu_{k l} V_{*}^{2}}{2 k_{B} T}\right) V_{*} \Theta\left(V_{*}-\frac{\Xi_{k l}}{c_{0}}\right)\left(\frac{\Xi_{k l}}{c_{0} V_{*}}\right)^{2} d \mathbf{V}_{*} \int_{\mathbb{R}^{3}} \exp \left(-\frac{M X^{2}}{2 k_{B} T}\right) d \mathbf{X}_{*} .
\end{aligned}
$$

Note that the integral with respect to $\mathbf{X}_{*}$ in the expression of $\mathcal{E}_{1 A}^{2}$ is null, so that $\mathcal{E}_{1 A}^{2}=0$. The integral with respect to $\mathbf{V}_{*}$ in the expression of $\mathcal{E}_{1 B}^{2}$ can be written in spherical coordinates and by writing $\mathbf{V}_{*}=V_{*} \overrightarrow{\mathbf{v}}$ with $\overrightarrow{\mathbf{v}}$ a unit vector, we can easily show that it vanishes, so $\mathcal{E}_{1 B}^{2}=0$. Thus $\mathcal{E}_{1}^{2}=0$. Since both $\mathcal{E}_{1}^{1}$ and $\mathcal{E}_{1}^{2}$ vanish, we have that $\mathcal{E}_{1}=0$. Similar computations show that $\mathcal{E}_{2}=0$. Putting all together we conclude that $\mathcal{E}=0$.

Now we prove that $\mathcal{F}$ given in 65 is equal to zero. As we have done above, we write it as the sum of the next two terms

$$
\begin{aligned}
& \mathcal{F}_{1}=-\sigma_{i j}^{2} \mathbf{a}_{i} \cdot \int_{\mathbb{R}^{3}} \int_{\mathbb{R}^{3}} \int_{\mathbb{S}_{+}^{2}} \mathbf{v}_{i} M_{i}^{\alpha} M_{j}^{\alpha} \Theta\left(\left\langle\epsilon, c_{0} \mathbf{v}_{i}-c_{0} \mathbf{v}_{j}\right\rangle-\Xi_{i j}\right)\left\langle\epsilon, \mathbf{v}_{i}-\mathbf{v}_{j}\right\rangle d \epsilon d \mathbf{v}_{j} d \mathbf{v}_{i}, \\
& \mathcal{F}_{2}=-\sigma_{i j}^{2} \mathbf{a}_{j} \cdot \int_{\mathbb{R}^{3}} \int_{\mathbb{R}^{3}} \int_{\mathbb{S}_{+}^{2}} \mathbf{v}_{j} M_{i}^{\alpha} M_{j}^{\alpha} \Theta\left(\left\langle\epsilon, c_{0} \mathbf{v}_{i}-c_{0} \mathbf{v}_{j}\right\rangle-\Xi_{i j}\right)\left\langle\epsilon, \mathbf{v}_{i}-\mathbf{v}_{j}\right\rangle d \epsilon d \mathbf{v}_{j} d \mathbf{v}_{i} .
\end{aligned}
$$

Evaluating the integral over $\mathbb{S}_{+}^{2}$ in $\mathcal{F}_{1}$ and $\mathcal{F}_{2}$ using $(67)$, we obtain

$$
\begin{aligned}
& \mathcal{F}_{1}=-\sigma_{i j}^{2} \mathbf{a}_{i} \cdot \int_{\mathbb{R}^{3}} \int_{\mathbb{R}^{3}} \mathbf{v}_{i} \exp \left[-\frac{m_{i}\left(\mathbf{v}_{i}\right)^{2}+m_{j}\left(\mathbf{v}_{j}\right)^{2}}{2 k_{B} T}\right] V \Theta\left(V-\frac{\Xi_{i j}}{c_{0}}\right)\left[1-\left(\frac{\Xi_{i j}}{c_{0} V}\right)^{2}\right] d \mathbf{v}_{j} d \mathbf{v}_{i} \\
& \mathcal{F}_{2}=-\sigma_{i j}^{2} \mathbf{a}_{j} \cdot \int_{\mathbb{R}^{3}} \int_{\mathbb{R}^{3}} \mathbf{v}_{j} \exp \left[-\frac{m_{i}\left(\mathbf{v}_{i}\right)^{2}+m_{j}\left(\mathbf{v}_{j}\right)^{2}}{2 k_{B} T}\right] V \Theta\left(V-\frac{\Xi_{i j}}{c_{0}}\right)\left[1-\left(\frac{\Xi_{i j}}{c_{0} V}\right)^{2}\right] d \mathbf{v}_{j} d \mathbf{v}_{i} .
\end{aligned}
$$

In order to show that $\mathcal{F}_{1}$ and $\mathcal{F}_{2}$ vanish it is enough to compute the above integrals. Upon expanding, we obtain similar expressions to those given in 89 , so that one can perform exactly the same computations as we did above to conclude that they both vanish. This shows that $\mathcal{F}=0$.

\subsection{Appendix B}

In this appendix, we compute the integrals $\mathcal{P}_{i}$ and $\mathcal{Q}_{i}$, that appear in 75 . Our first goal is to show that

$$
\mathcal{P}_{i}=\frac{8}{9} \frac{\mu_{i j}}{k_{B} T} \alpha^{2} \sigma_{i j}^{2} \sqrt{\pi} \frac{m_{j}}{M} c_{i}^{\alpha} c_{j}^{\alpha}\left(\mathbf{u}_{j}^{\alpha}-\mathbf{u}_{i}^{\alpha}\right)\left[\left(\frac{2 k_{B} T}{\mu_{i j}}\right)^{\frac{3}{2}} \Gamma\left(3, z_{i}^{*}\right)-\left(\frac{\Xi_{i j}}{c_{0}}\right)^{3} \Gamma\left(\frac{3}{2}, z_{i}^{*}\right)\right] .
$$

Using Lemma 3.9 with $\varphi\left(\mathbf{v}_{i}^{\prime}\right)=\mathbf{v}_{i}^{\prime}$ and $\varphi\left(\mathbf{v}_{i}\right)=\mathbf{v}_{i}$, we obtain

$$
\mathcal{P}_{i}=\sigma_{i j}^{2} \alpha \int_{\mathbb{R}^{3}} \int_{\mathbb{R}^{3}} \int_{\mathbb{S}_{+}^{2}}\left(\mathbf{v}_{i}^{\prime}-\mathbf{v}_{i}\right) f_{i} f_{j} \Theta\left(\left\langle\epsilon, \mathbf{v}_{i}-\mathbf{v}_{j}\right\rangle-\Xi_{i j}\right)\left\langle\epsilon, \mathbf{v}_{i}-\mathbf{v}_{j}\right\rangle d \epsilon d \mathbf{v}_{j} d \mathbf{v}_{i} .
$$

Using the first expression in $(14)$ for $\mathbf{v}_{i}^{\prime}$ and writing the integral over $\mathbb{S}_{+}^{2}$ in spherical coordinates as described in Remark 3.1, where in both cases $s$ is replaced by $j$, we obtain 


$$
\begin{aligned}
\mathcal{P}_{i}=2 \sigma_{i j}^{2} \alpha \frac{m_{j}}{M} & \\
& \times \int_{\mathbb{R}^{3}} \int_{\mathbb{R}^{3}} \int_{0}^{\frac{\pi}{2}} \int_{0}^{2 \pi}\left(\mathbf{v}_{j}-\mathbf{v}_{i}\right) f_{i}^{\alpha} f_{j}^{\alpha} \Theta\left(\left\langle\epsilon, c_{0} \mathbf{v}_{i}-c_{0} \mathbf{v}_{j}\right\rangle-\Xi_{i j}\right) V \cos ^{2} \theta \sin \theta d \phi d \theta d \mathbf{v}_{j} d \mathbf{v}_{i} .
\end{aligned}
$$

Integrating with respect to $\phi$ and observing that

$$
\int_{0}^{\arccos \left(\frac{\Xi_{i j}}{c_{0} V}\right)} \cos ^{2} \theta \sin \theta d \theta=\frac{1}{3}\left[1-\left(\frac{\Xi_{i j}}{c_{0} V}\right)^{3}\right]
$$

we get

$$
\mathcal{P}_{i}=\sigma_{i j}^{2} \alpha \frac{4 \pi}{3} \frac{m_{j}}{M} \int_{\mathbb{R}^{3}} \int_{\mathbb{R}^{3}} \mathbf{V} f_{i}^{\alpha} f_{j}^{\alpha} \Theta\left(V-\frac{\Xi_{i j}}{c_{0}}\right) V\left[1-\left(\frac{\Xi_{i j}}{c_{0} V}\right)^{3}\right] d \mathbf{v}_{j} d \mathbf{v}_{i},
$$

where $\mathbf{V}=\mathbf{v}_{j}-\mathbf{v}_{i}$ is the relative velocity and $V=\left\|\mathbf{v}_{j}-\mathbf{v}_{i}\right\|$. Now, we can split $\mathcal{P}_{i}$ into

$$
\begin{aligned}
\mathcal{P}_{i} & =\underbrace{\sigma_{i j}^{2} \alpha \frac{4 \pi}{3} \frac{m_{j}}{M} \int_{\mathbb{R}^{3}} \int_{\mathbb{R}^{3}} \mathbf{V} f_{i}^{\alpha} f_{j}^{\alpha} \Theta\left(V-\frac{\Xi_{i j}}{c_{0}}\right) V d \mathbf{v}_{j} d \mathbf{v}_{i}}_{\mathcal{P}_{i_{1}}} \\
& -\underbrace{\sigma_{i j}^{2} \alpha \frac{4 \pi}{3} \frac{m_{j}}{M} \int_{\mathbb{R}^{3}} \int_{\mathbb{R}^{3}} \mathbf{V} f_{i}^{\alpha} f_{j}^{\alpha} \Theta\left(V-\frac{\Xi_{i j}}{c_{0}}\right) V\left(\frac{\Xi_{i j}}{c_{0} V}\right)^{3} d \mathbf{v}_{j} d \mathbf{v}_{i}}_{\mathcal{P}_{i_{2}}} .
\end{aligned}
$$

Using [64), neglecting the quadratic term in the coefficient $\mathbf{a}$ and transforming from $\mathbf{v}_{i}$ and $\mathbf{v}_{j}$ to the centre of mass velocity $\mathbf{X}$ and relative velocity $\mathbf{V}$ and also using the fact that the Jacobian of the transformation has absolute value 1 , we obtain

$$
\begin{aligned}
& \mathcal{P}_{i_{1}}=\frac{4 \pi}{3} \sigma_{i j}^{2} \alpha \frac{m_{j}}{M} c_{i}^{\alpha} c_{j}^{\alpha} \frac{\left(m_{i} m_{j}\right)^{\frac{3}{2}}}{\left(2 \pi k_{B} T\right)^{3}} \int_{\mathbb{R}^{3}} \int_{\mathbb{R}^{3}} \mathbf{V} \exp \left(-\frac{M X^{2}+\mu_{i j} V^{2}}{2 k_{B} T}\right) V \Theta\left(V-\frac{\Xi_{i j}}{c_{0}}\right) \\
& \times\left[1+\left(\mathbf{a}_{j}+\mathbf{a}_{i}\right) \cdot \mathbf{X}+\left(\frac{\mathbf{a}_{j} m_{i}-\mathbf{a}_{i} m_{j}}{M}\right) \cdot \mathbf{V}\right] d \mathbf{X} d \mathbf{V}, \\
& \mathcal{P}_{i_{2}}=\frac{4 \pi}{3} \sigma_{i j}^{2} \alpha \frac{m_{j}}{M} c_{i}^{\alpha} c_{j}^{\alpha} \frac{\left(m_{i} m_{j}\right)^{\frac{3}{2}}}{\left(2 \pi k_{B} T\right)^{3}} \int_{\mathbb{R}^{3}} \int_{\mathbb{R}^{3}} \mathbf{V} \exp \left(-\frac{M X^{2}+\mu_{i j} V^{2}}{2 k_{B} T}\right) \Theta\left(V-\frac{\Xi_{i j}}{c_{0}}\right) V\left(\frac{\Xi_{i j}}{c_{0} V}\right)^{3} \\
& \times\left[1+\left(\mathbf{a}_{j}+\mathbf{a}_{i}\right) \cdot \mathbf{X}+\left(\frac{\mathbf{a}_{j} m_{i}-\mathbf{a}_{i} m_{j}}{M}\right) \cdot \mathbf{V}\right] d \mathbf{X} d \mathbf{V} .
\end{aligned}
$$

We can split the integral appearing in $\mathcal{P}_{i_{1}}$ as the sum of

$$
\begin{aligned}
& \mathcal{P}_{i_{1}}^{1}=\int_{\mathbb{R}^{3}} \exp \left(-\frac{M X^{2}}{2 k_{B} T}\right) d \mathbf{X} \int_{\mathbb{R}^{3}} \mathbf{V} \exp \left(-\frac{\mu_{i j} V^{2}}{2 k_{B} T}\right) V \Theta\left(V-\frac{\Xi_{i j}}{c_{0}}\right) d \mathbf{V} \\
& \mathcal{P}_{i_{1}}^{2}=\left(\mathbf{a}_{j}+\mathbf{a}_{i}\right) \cdot \int_{\mathbb{R}^{3}} \mathbf{X} \exp \left(-\frac{M X^{2}}{2 k_{B} T}\right) d \mathbf{X} \int_{\mathbb{R}^{3}} \mathbf{V} \exp \left(-\frac{\mu_{i j} V^{2}}{2 k_{B} T}\right) V \Theta\left(V-\frac{\Xi_{i j}}{c_{0}}\right) d \mathbf{V} \\
& \mathcal{P}_{i_{1}}^{3}=\frac{m_{i}}{M} \int_{\mathbb{R}^{3}} \exp \left(-\frac{M X^{2}}{2 k_{B} T}\right) d \mathbf{X} \int_{\mathbb{R}^{3}}\left(\mathbf{a}_{j} \cdot \mathbf{V}\right) \mathbf{V} \exp \left(-\frac{\mu_{i j} V^{2}}{2 k_{B} T}\right) V \Theta\left(V-\frac{\Xi_{i j}}{c_{0}}\right) d \mathbf{V} \\
& \mathcal{P}_{i_{1}}^{4}=-\frac{m_{j}}{M} \int_{\mathbb{R}^{3}} \exp \left(-\frac{M X^{2}}{2 k_{B} T}\right) d \mathbf{X} \int_{\mathbb{R}^{3}}\left(\mathbf{a}_{i} \cdot \mathbf{V}\right) \mathbf{V} \exp \left(-\frac{\mu_{i j} V^{2}}{2 k_{B} T}\right) V \Theta\left(V-\frac{\Xi_{i j}}{c_{0}}\right) d \mathbf{V} .
\end{aligned}
$$

Observe that the integrals with respect to $\mathbf{V}$ in both $\mathcal{P}_{i_{1}}^{1}$ and $\mathcal{P}_{i_{1}}^{2}$ are equal. By using the fact that $\mathbf{V}=V \overrightarrow{\mathbf{v}}$ with $\overrightarrow{\mathbf{v}}$ a unit vector and $V=\|\mathbf{V}\|$, we note that writing them in spherical coordinates, it is easy to see that 
they both vanish. Therefore, $\mathcal{P}_{i_{1}}^{1}=\mathcal{P}_{i_{1}}^{2}=0$. The integral with respect to $\mathbf{X}$ appearing in $\mathcal{P}_{i_{1}}^{3}$ and $\mathcal{P}_{i_{1}}^{4}$ has been computed in 699). By setting $\left(\mathbf{a}_{j} \cdot \mathbf{V}\right) \mathbf{V}=a_{j} \cos \theta V^{2} \overrightarrow{\mathbf{v}}$, the integral with respect to $\mathbf{V}$ in $\mathcal{P}_{i_{1}}^{3}$ can be written in spherical coordinates as given below

$$
\begin{aligned}
\int_{\mathbb{R}^{3}} a_{j} \cos \theta V^{2} \overrightarrow{\mathbf{v}} & \exp \left(-\frac{\mu_{i j} V^{2}}{2 k_{B} T}\right) V \Theta\left(V-\frac{\Xi_{i j}}{c_{0}}\right) d \mathbf{V} \\
= & a_{j} \int_{V \geq \frac{\Xi_{i j}}{c_{0}}}^{\infty} V^{5} \exp \left(-\frac{\mu_{i j} V^{2}}{2 k_{B} T}\right) d V(\hat{\mathbf{x}} \int_{0}^{\pi} \sin ^{2} \theta \cos \theta d \theta \underbrace{\int_{0}^{2 \pi} \cos \phi d \phi}_{0} \\
& +\hat{\mathbf{y}} \int_{0}^{\pi} \sin ^{2} \theta \cos \theta d \theta \underbrace{\int_{0}^{2 \pi} \sin \phi d \phi}_{0}+\hat{\mathbf{z}} \underbrace{\int_{0}^{\pi} \sin \theta \cos ^{2} \theta d \theta}_{\frac{2}{3}} \underbrace{\int_{0}^{2 \pi} d \phi}_{2 \pi}),
\end{aligned}
$$

where $\hat{\mathbf{x}}, \hat{\mathbf{y}}, \hat{\mathbf{z}}$ are the Cartesian unit vectors in $\mathbb{R}^{3}$. Using the transformation $z=\frac{\mu_{i j}}{2 k_{B} T} V^{2}$ together with 59 , we obtain

$$
\int_{V \geq \frac{\Xi_{i j}}{c_{0}}}^{\infty} V^{5} \exp \left(-\frac{\mu_{i j} V^{2}}{2 k_{B} T}\right) d V=\frac{1}{2}\left(\frac{2 k_{B} T}{\mu_{i j}}\right)^{3} \Gamma\left(3, z_{i}^{*}\right),
$$

where $z_{i}^{*}$ is defined as in 62 . Therefore,

$$
\int_{\mathbb{R}^{3}}\left(\mathbf{a}_{j} \cdot \mathbf{V}\right) \mathbf{V} \exp \left(-\frac{\mu_{i j} V^{2}}{2 k_{B} T}\right) V \Theta\left(V-\frac{\Xi_{i j}}{c_{0}}\right) d \mathbf{V}=\frac{2 \pi}{3}\left(\frac{2 k_{B} T}{\mu_{i j}}\right)^{3} \Gamma\left(3, z_{i}^{*}\right) \mathbf{a}_{j} .
$$

Similarly,

$$
\int_{\mathbb{R}^{3}}\left(\mathbf{a}_{i} \cdot \mathbf{V}\right) \mathbf{V} \exp \left(-\frac{\mu_{i j} V^{2}}{2 k_{B} T}\right) V \Theta\left(V-\frac{\Xi_{i j}}{c_{0}}\right) d \mathbf{V}=\frac{2 \pi}{3}\left(\frac{2 k_{B} T}{\mu_{i j}}\right)^{3} \Gamma\left(3, z_{i}^{*}\right) \mathbf{a}_{i} .
$$

Putting together the previous computations and doing some algebra, we conclude that

$$
\mathcal{P}_{i_{1}}=\frac{8 \sqrt{\pi}}{9} \sigma_{i j}^{2} \alpha^{2} \frac{m_{j}}{M} c_{i}^{\alpha} c_{j}^{\alpha} \frac{\mu_{i j}}{k_{B} T}\left(\mathbf{u}_{j}^{\alpha}-\mathbf{u}_{i}^{\alpha}\right)\left(\frac{2 k_{B} T}{\mu_{i j}}\right)^{\frac{3}{2}} \Gamma\left(3, z_{i}^{*}\right) .
$$

Now we compute $\mathcal{P}_{i_{2}}$ by repeating exactly the same computations as we did for $\mathcal{P}_{i_{1}}$. Splitting $\mathcal{P}_{i_{2}}$ into four terms, we see that two of them vanish by the same reason as above and the remaining two are as given below

$$
\begin{aligned}
\mathcal{P}_{i_{2}}^{3}= & \frac{4 \pi}{3} \sigma_{i j}^{2} \alpha \frac{m_{j}}{M} c_{i}^{\alpha} c_{j}^{\alpha} \frac{\left(m_{i} m_{j}\right)^{\frac{3}{2}}}{\left(2 \pi k_{B} T^{3}\right.} \frac{m_{i}}{M}\left(\frac{\Xi_{i j}}{c_{0}}\right)^{3} \\
& \times \int_{\mathbb{R}^{3}} \exp \left(-\frac{M X^{2}}{2 k_{B} T}\right) d \mathbf{X} \int_{\mathbb{R}^{3}}\left(\mathbf{a}_{j} \cdot \mathbf{V}\right) \mathbf{V} \exp \left(-\frac{\mu_{i j} V^{2}}{2 k_{B} T}\right) \Theta\left(V-\frac{\Xi_{i j}}{c_{0}}\right) d \mathbf{V}, \\
\mathcal{P}_{i_{2}}^{4}= & -\frac{4 \pi}{3} \sigma_{i j}^{2} \alpha \frac{m_{j}}{M} c_{i}^{\alpha} c_{j}^{\alpha} \frac{\left(m_{i} m_{j}\right)^{\frac{3}{2}}}{\left(2 \pi k_{B} T^{3}\right.} \frac{m_{j}}{M}\left(\frac{\Xi_{i j}}{c_{0}}\right)^{3} \\
& \times \int_{\mathbb{R}^{3}} \exp \left(-\frac{M X^{2}}{2 k_{B} T}\right) d \mathbf{X} \int_{\mathbb{R}^{3}}\left(\mathbf{a}_{i} \cdot \mathbf{V}\right) \mathbf{V} \exp \left(-\frac{\mu_{i j} V^{2}}{2 k_{B} T}\right) \Theta\left(V-\frac{\Xi_{i j}}{c_{0}}\right) d \mathbf{V} .
\end{aligned}
$$

The integral with respect to $\mathbf{X}$ appearing in $\mathcal{P}_{i_{2}}^{3}$ and $\mathcal{P}_{i_{2}}^{4}$ has been computed in 69 and the integrals with respect to $\mathbf{V}$ can be computed as we did above to obtain

$$
\left(\frac{\Xi_{i j}}{c_{0}}\right)^{3}\left(\frac{2 k_{B} T}{\mu_{i j}}\right)^{\frac{3}{2}} \frac{2 \pi}{3} \Gamma\left(\frac{3}{2}, z_{i}^{*}\right) \mathbf{a}_{j} \quad \text { and } \quad\left(\frac{\Xi_{i j}}{c_{0}}\right)^{3}\left(\frac{2 k_{B} T}{\mu_{i j}}\right)^{\frac{3}{2}} \frac{2 \pi}{3} \Gamma\left(\frac{3}{2}, z_{i}^{*}\right) \mathbf{a}_{i} .
$$


Putting together the previous computations and doing some algebra, we conclude that

$$
\mathcal{P}_{i_{2}}=\frac{8 \sqrt{\pi}}{9} \sigma_{i j}^{2} \alpha^{2} \frac{m_{j}}{M} c_{i}^{\alpha} c_{j}^{\alpha} \frac{\mu_{i j}}{k_{B} T}\left(\mathbf{u}_{j}^{\alpha}-\mathbf{u}_{i}^{\alpha}\right)\left(\frac{\Xi_{i j}}{c_{0}}\right)^{3} \Gamma\left(\frac{3}{2}, z_{i}^{*}\right) .
$$

Substituting (94), 95) into (92) and factorizing gives the desired expression (91).

The last term on the right hand side of $(73)$, that is the integral $\mathcal{Q}_{i}$ given in $(75)$, can be written as the sum of the next three terms, where quadratic terms in the coefficients a have been neglected,

$$
\begin{aligned}
& \mathcal{R}=\alpha \sigma_{i j}^{2} \int_{\mathbb{R}^{3}} \int_{\mathbb{R}^{3}} \int_{\mathbb{S}_{+}^{2}}\left(\left(\frac{\mu_{i j}}{\mu_{k l}}\right)^{2} M_{k}^{\alpha \circ} M_{l}^{\alpha \circ}-M_{i}^{\alpha} M_{j}^{\alpha}\right) \mathbf{v}_{i} \\
& \quad \times \Theta\left(\left\langle\epsilon, c_{0} \mathbf{v}_{i}-c_{0} \mathbf{v}_{j}\right\rangle-\Xi_{i j}\right)\left\langle\epsilon, \mathbf{v}_{i}-\mathbf{v}_{j}\right\rangle d \epsilon d \mathbf{v}_{j} d \mathbf{v}_{i}, \\
& \mathcal{S}=\alpha \sigma_{i j}^{2} \int_{\mathbb{R}^{3}} \int_{\mathbb{R}^{3}} \int_{\mathbb{S}_{+}^{2}}\left(\frac{\mu_{i j}}{\mu_{k l}}\right)^{2} M_{k}^{\alpha \circ} M_{l}^{\alpha \circ}\left(\mathbf{a}_{k} \cdot \mathbf{v}_{k}^{\circ}+\mathbf{a}_{l} \cdot \mathbf{v}_{l}^{\circ}\right) \mathbf{v}_{i} \\
& \times \Theta\left(\left\langle\epsilon, c_{0} \mathbf{v}_{i}-c_{0} \mathbf{v}_{j}\right\rangle-\Xi_{i j}\right)\left\langle\epsilon, \mathbf{v}_{i}-\mathbf{v}_{j}\right\rangle d \epsilon d \mathbf{v}_{j} d \mathbf{v}_{i}, \\
& \mathcal{T}=-\alpha \sigma_{i j}^{2} \int_{\mathbb{R}^{3}} \int_{\mathbb{R}^{3}} \int_{\mathbb{S}_{+}^{2}} M_{i}^{\alpha} M_{j}^{\alpha}\left(\mathbf{a}_{i} \cdot \mathbf{v}_{i}+\mathbf{a}_{j} \cdot \mathbf{v}_{j}\right) \mathbf{v}_{i} \Theta\left(\left\langle\epsilon, c_{0} \mathbf{v}_{i}-c_{0} \mathbf{v}_{j}\right\rangle-\Xi_{i j}\right)\left\langle\epsilon, \mathbf{v}_{i}-\mathbf{v}_{j}\right\rangle d \epsilon d \mathbf{v}_{j} d \mathbf{v}_{i} .
\end{aligned}
$$

First we prove that $\mathcal{R}=0$. Observe that $\mathcal{R}$ is similar to the integral $\mathcal{D}$ given in $(65)$ and, therefore, writing $\mathcal{R}$ in terms of centre of mass velocity $\mathbf{X}$ and relative velocity $\mathbf{V}$, and expanding the resulting integral, we realize that $\mathcal{R}$ can be written as the difference of the two next terms

$$
\begin{aligned}
& \mathcal{R}_{1}=\Delta \int_{\mathbb{R}^{3}} \mathbf{X} \exp \left(-\frac{M X^{2}}{2 k_{B} T}\right) d \mathbf{X} \int_{\mathbb{R}^{3}} V \Theta\left(V-\frac{\Xi_{i j}}{c_{0}}\right)\left[1-\left(\frac{\Xi_{i j}}{c_{0} V}\right)^{2}\right] \exp \left(-\frac{\mu_{i j} V^{2}}{2 k_{B} T}\right) d \mathbf{V} \\
& \mathcal{R}_{2}=\frac{m_{j}}{M} \Delta \int_{\mathbb{R}^{3}} \exp \left(-\frac{M X^{2}}{2 k_{B} T}\right) d \mathbf{X} \int_{\mathbb{R}^{3}} \mathbf{V} V \Theta\left(V-\frac{\Xi_{i j}}{c_{0}}\right)\left[1-\left(\frac{\Xi_{i j}}{c_{0} V}\right)^{2}\right] \exp \left(-\frac{\mu_{i j} V^{2}}{2 k_{B} T}\right) d \mathbf{V}
\end{aligned}
$$

where

$$
\Delta=\pi \alpha \sigma_{i j}^{2} \frac{\left(m_{i} m_{j}\right)^{\frac{3}{2}}}{\left(2 \pi k_{B} T\right)^{3}}\left[\left(\frac{\mu_{i j}}{\mu_{k l}}\right)^{\frac{1}{2}} c_{k}^{\alpha} c_{l}^{\alpha} e^{\frac{Q_{R}}{k_{B} T}}-c_{i}^{\alpha} c_{j}^{\alpha}\right] .
$$

Since the integral with respect to $\mathbf{X}$ in $\mathcal{R}_{1}$ is zero, we conclude that the contribution to $\mathcal{R}$ comes only from $\mathcal{R}_{2}$, which can be rewritten as

$$
\begin{aligned}
& \frac{m_{j}}{M} \Delta \int_{\mathbb{R}^{3}} \exp \left(-\frac{M X^{2}}{2 k_{B} T}\right) d \mathbf{X} \int_{\mathbb{R}^{3}} \mathbf{V e x p}\left(-\frac{\mu_{i j} V^{2}}{2 k_{B} T}\right) V \Theta\left(V-\frac{\Xi_{i j}}{c_{0}}\right) d \mathbf{V} \\
& \quad-\frac{m_{j}}{M} \Delta \int_{\mathbb{R}^{3}} \exp \left(-\frac{M X^{2}}{2 k_{B} T}\right) d \mathbf{X} \int_{\mathbb{R}^{3}} \mathbf{V}\left(\frac{\Xi_{i j}}{c_{0} V}\right)^{2} \exp \left(-\frac{\mu_{i j} V^{2}}{2 k_{B} T}\right) V \Theta\left(V-\frac{\Xi_{i j}}{c_{0}}\right) d \mathbf{V} .
\end{aligned}
$$

Transforming both integrals in $\mathbf{V}$ above to spherical coordinates and using the fact that $\mathbf{V}=V \overrightarrow{\mathbf{v}}$ with $\overrightarrow{\mathbf{v}}$ a unit vector and $V=\|\mathbf{V}\|$, we get that both integrals vanish. This shows that $\mathcal{R}=0$ as desired. Now we analyze $\mathcal{S}$, which can be written as the sum of the next two terms

$$
\begin{aligned}
& \mathcal{S}_{1}=\alpha \sigma_{i j}^{2}\left(\frac{\mu_{i j}}{\mu_{k l}}\right)^{2} \int_{\mathbb{R}^{3}} \int_{\mathbb{R}^{3}} \int_{\mathbb{S}_{+}^{2}}\left(\mathbf{a}_{k} \cdot \mathbf{v}_{k}^{\circ}\right) \mathbf{v}_{i} M_{k}^{\alpha} M_{l}^{\alpha} \Theta\left(\left\langle\epsilon, c_{0} \mathbf{v}_{i}-c_{0} \mathbf{v}_{j}\right\rangle-\Xi_{i j}\right)\left\langle\epsilon, \mathbf{v}_{i}-\mathbf{v}_{j}\right\rangle d \epsilon d \mathbf{v}_{j} d \mathbf{v}_{i} \\
& \mathcal{S}_{2}=\alpha \sigma_{i j}^{2}\left(\frac{\mu_{i j}}{\mu_{k l}}\right)^{2} \int_{\mathbb{R}^{3}} \int_{\mathbb{R}^{3}} \int_{\mathbb{S}_{+}^{2}}\left(\mathbf{a}_{l} \cdot \mathbf{v}_{l}^{\circ}\right) \mathbf{v}_{i} M_{k}^{\alpha} M_{l}^{\alpha} \Theta\left(\left\langle\epsilon, c_{0} \mathbf{v}_{i}-c_{0} \mathbf{v}_{j}\right\rangle-\Xi_{i j}\right)\left\langle\epsilon, \mathbf{v}_{i}-\mathbf{v}_{j}\right\rangle d \epsilon d \mathbf{v}_{j} d \mathbf{v}_{i} .
\end{aligned}
$$


Now we change variables from $\mathbf{v}_{i}, \mathbf{v}_{j}$ to $\mathbf{v}_{k}^{\circ}, \mathbf{v}_{l}^{\circ}$, by using the first expression of (26) given in Property 3.4 together with 25) to rewrite $\mathcal{S}_{1}$ and $\mathcal{S}_{2}$ as

$$
\begin{aligned}
& \mathcal{S}_{1}=\alpha \sigma_{i j}^{2}\left(\frac{\mu_{i j}}{\mu_{k l}}\right)^{2} \int_{\mathbb{R}^{3}} \int_{\mathbb{R}^{3}} \int_{\mathbb{S}_{+}^{2}}\left(\mathbf{a}_{k} \cdot \mathbf{v}_{k}^{\circ}\right) \mathbf{v}_{k}^{\circ} M_{k}^{\alpha} M_{l}^{\alpha} \Theta\left(\left\langle\epsilon, c_{0} \mathbf{v}_{k}^{\circ}-c_{0} \mathbf{v}_{l}^{\circ}\right\rangle-\Xi_{k l}\right) \\
& \times\left(\frac{\mu_{k l}}{\mu_{i j}}\right)^{\frac{1}{2}} \omega^{+}\left(\frac{\mu_{k l}}{\mu_{i j}}\right)^{\frac{3}{2}} \frac{\left\langle\epsilon, \mathbf{v}_{k}^{\circ}-\mathbf{v}_{l}^{\circ}\right\rangle}{\omega^{+}} d \epsilon d \mathbf{v}_{k}^{\circ} d \mathbf{v}_{l}^{\circ}, \\
& \mathcal{S}_{2}=\alpha \sigma_{i j}^{2}\left(\frac{\mu_{i j}}{\mu_{k l}}\right)^{2} \int_{\mathbb{R}^{3}} \int_{\mathbb{R}^{3}} \int_{\mathbb{S}_{+}^{2}}\left(\mathbf{a}_{l} \cdot \mathbf{v}_{l}^{\circ}\right) \mathbf{v}_{k}^{\circ} M_{k}^{\alpha} M_{l}^{\alpha} \Theta\left(\left\langle\epsilon, c_{0} \mathbf{v}_{k}^{\circ}-c_{0} \mathbf{v}_{l}^{\circ}\right\rangle-\Xi_{k l}\right) \\
& \times\left(\frac{\mu_{k l}}{\mu_{i j}}\right)^{\frac{1}{2}} \omega^{+}\left(\frac{\mu_{k l}}{\mu_{i j}}\right)^{\frac{3}{2}} \frac{\left\langle\epsilon, \mathbf{v}_{k}^{\circ}-\mathbf{v}_{l}^{\circ}\right\rangle}{\omega^{+}} d \epsilon d \mathbf{v}_{k}^{\circ} d \mathbf{v}_{l}^{\circ} .
\end{aligned}
$$

Therefore,

$$
\begin{aligned}
& \mathcal{S}_{1}=\sigma_{i j}^{2} \alpha \int_{\mathbb{R}^{3}} \int_{\mathbb{R}^{3}} \int_{\mathbb{S}_{+}^{2}}\left(\mathbf{a}_{k} \cdot \mathbf{v}_{k}^{\circ}\right) \mathbf{v}_{k}^{\circ} M_{k}^{\alpha \circ} M_{l}^{\alpha \circ} \Theta\left(\left\langle\epsilon, c_{0} \mathbf{v}_{k}^{\circ}-c_{0} \mathbf{v}_{l}^{\circ}\right\rangle-\Xi_{k l}\right)\left\langle\epsilon, \mathbf{v}_{k}^{\circ}-\mathbf{v}_{l}^{\circ}\right\rangle d \epsilon d \mathbf{v}_{k}^{\circ} d \mathbf{v}_{l}^{\circ} \\
& \mathcal{S}_{2}=\sigma_{i j}^{2} \alpha \int_{\mathbb{R}^{3}} \int_{\mathbb{R}^{3}} \int_{\mathbb{S}_{+}^{2}}\left(\mathbf{a}_{l} \cdot \mathbf{v}_{l}^{\circ}\right) \mathbf{v}_{k}^{\circ} M_{k}^{\alpha \circ} M_{l}^{\alpha \circ} \Theta\left(\left\langle\epsilon, c_{0} \mathbf{v}_{k}^{\circ}-c_{0} \mathbf{v}_{l}^{\circ}\right\rangle-\Xi_{k l}\right)\left\langle\epsilon, \mathbf{v}_{k}^{\circ}-\mathbf{v}_{l}^{\circ}\right\rangle d \epsilon d \mathbf{v}_{k}^{\circ} d \mathbf{v}_{l}^{\circ}
\end{aligned}
$$

Performing similar computations to those of 67), we obtain

$$
\begin{aligned}
& \mathcal{S}_{1}=\sigma_{i j}^{2} \alpha \pi \int_{\mathbb{R}^{3}} \int_{\mathbb{R}^{3}}\left(\mathbf{a}_{k} \cdot \mathbf{v}_{k}^{\circ}\right) \mathbf{v}_{k}^{\circ} M_{k}^{\alpha \circ} M_{l}^{\alpha \circ} V_{*} \Theta\left(V_{*}-\frac{\Xi_{k l}}{c_{0}}\right)\left[1-\left(\frac{\Xi_{k l}}{c_{0} V_{*}}\right)^{2}\right] d \mathbf{v}_{k}^{\circ} d \mathbf{v}_{l}^{\circ}, \\
& \mathcal{S}_{2}=\sigma_{i j}^{2} \alpha \pi \int_{\mathbb{R}^{3}} \int_{\mathbb{R}^{3}}\left(\mathbf{a}_{l} \cdot \mathbf{v}_{l}^{\circ}\right) \mathbf{v}_{k}^{\circ} M_{k}^{\alpha \circ} M_{l}^{\alpha \circ} V_{*} \Theta\left(V_{*}-\frac{\Xi_{k l}}{c_{0}}\right)\left[1-\left(\frac{\Xi_{k l}}{c_{0} V_{*}}\right)^{2}\right] d \mathbf{v}_{k}^{\circ} d \mathbf{v}_{l}^{\circ},
\end{aligned}
$$

where $V_{*}=\left\|\mathbf{v}_{k}^{\circ}-\mathbf{v}_{l}^{\circ}\right\|=\left\|\mathbf{v}_{l}^{\circ}-\mathbf{v}_{k}^{\circ}\right\|$.

Now, expanding $\mathcal{S}_{1}$ and transforming from $\mathbf{v}_{k}^{\circ}$ and $\mathbf{v}_{l}^{\circ}$ to the centre of mass velocity $\mathbf{X}_{*}=\left(m_{k} \mathbf{v}_{k}^{\circ}+m_{l} \mathbf{v}_{l}^{\circ}\right) / M$ and relative velocity $\mathbf{V}_{*}=\mathbf{v}_{l}^{\circ}-\mathbf{v}_{k}^{\circ}$, as well as using the fact that the Jacobian of the transformation has absolute value 1 , we obtain that it can be written as the difference of the next two terms

$$
\begin{aligned}
& \mathcal{S}_{1}^{1}=\sigma_{i j}^{2} \alpha \pi c_{k}^{\alpha} c_{l}^{\alpha} \frac{\left(m_{k} m_{l}\right)^{\frac{3}{2}}}{\left(2 \pi k_{B} T\right)^{3}} \int_{\mathbb{R}^{3}} \int_{\mathbb{R}^{3}}\left(\mathbf{X}_{*}-\frac{m_{l} \mathbf{V}_{*}}{m_{k}+m_{l}}\right)\left[\mathbf{a}_{k} \cdot\left(\mathbf{X}_{*}-\frac{m_{l} \mathbf{V}_{*}}{m_{k}+m_{l}}\right)\right] \\
& \times \exp \left(-\frac{M X_{*}^{2}+\mu_{k l} V_{*}^{2}}{2 k_{B} T}\right) V_{*} \Theta\left(V_{*}-\frac{\Xi_{k l}}{c_{0}}\right) d \mathbf{X}_{*} d \mathbf{V}_{*}, \\
& \mathcal{S}_{1}^{2}=\sigma_{i j}^{2} \alpha \pi c_{k}^{\alpha} c_{l}^{\alpha} \frac{\left(m_{k} m_{l}\right)^{\frac{3}{2}}}{\left(2 \pi k_{B} T\right)^{3}} \int_{\mathbb{R}^{3}} \int_{\mathbb{R}^{3}}\left(\mathbf{X}_{*}-\frac{m_{l} \mathbf{V}_{*}}{m_{k}+m_{l}}\right)\left[\mathbf{a}_{k} \cdot\left(\mathbf{X}_{*}-\frac{m_{l} \mathbf{V}_{*}}{m_{k}+m_{l}}\right)\right] \\
& \times \exp \left(-\frac{M X_{*}^{2}+\mu_{k l} V_{*}^{2}}{2 k_{B} T}\right) V_{*} \Theta\left(V_{*}-\frac{\Xi_{k l}}{c_{0}}\right)\left(\frac{\Xi_{k l}}{c_{0} V_{*}}\right)^{2} d \mathbf{X}_{*} d \mathbf{V}_{*},
\end{aligned}
$$

where we have used (58). Expanding $\mathcal{S}_{1}^{1}$, we obtain

$$
\mathcal{S}_{1}^{1}=\sigma_{i j}^{2} \alpha \pi c_{k}^{\alpha} c_{l}^{\alpha} \frac{\left(m_{k} m_{l}\right)^{\frac{3}{2}}}{\left(2 \pi k_{B} T\right)^{3}} \int_{\mathbb{R}^{3}}\left(\mathbf{a}_{k} \cdot \mathbf{X}_{*}\right) \mathbf{X}_{*} \exp \left(-\frac{M X_{*}^{2}}{2 k_{B} T}\right) d \mathbf{X}_{*}
$$




$$
\begin{array}{r}
\times \int_{\mathbb{R}^{3}} \exp \left(-\frac{\mu_{k l} V_{*}^{2}}{2 k_{B} T}\right) V_{*} \Theta\left(V_{*}-\frac{\Xi_{k l}}{c_{0}}\right) d \mathbf{V}_{*} \\
-\sigma_{i j}^{2} \alpha \pi c_{k}^{\alpha} c_{l}^{\alpha} \frac{\left(m_{k} m_{l}\right)^{\frac{3}{2}}}{\left(2 \pi k_{B} T\right)^{3}} \frac{m_{l}}{m_{k}+m_{l}} \int_{\mathbb{R}^{3}} \mathbf{X}_{*} \exp \left(-\frac{M X_{*}^{2}}{2 k_{B} T}\right) d \mathbf{X}_{*} \\
\times \mathbf{a}_{k} \cdot \int_{\mathbb{R}^{3}} \mathbf{V}_{*} \exp \left(-\frac{\mu_{k l} V_{*}^{2}}{2 k_{B} T}\right) V_{*} \Theta\left(V_{*}-\frac{\Xi_{k l}}{c_{0}}\right) d \mathbf{V}_{*} \\
-\sigma_{i j}^{2} \alpha \pi c_{k}^{\alpha} c_{l}^{\alpha} \frac{\left(m_{k} m_{l}\right)^{\frac{3}{2}}}{\left(2 \pi k_{B} T\right)^{3}} \frac{m_{l}}{m_{k}+m_{l}} \mathbf{a}_{k} \cdot \int_{\mathbb{R}^{3}} \mathbf{X}_{*} \exp \left(-\frac{M X_{*}^{2}}{2 k_{B} T}\right) d \mathbf{X}_{*} \\
\times \int_{\mathbb{R}^{3}} \mathbf{V}_{*} \exp \left(-\frac{\mu_{k l} V_{*}^{2}}{2 k_{B} T}\right) V_{*} \Theta\left(V_{*}-\frac{\Xi_{k l}}{c_{0}}\right) d \mathbf{V}_{*} \\
+\sigma_{i j}^{2} \alpha \pi c_{k}^{\alpha} c_{l}^{\alpha} \frac{\left(m_{k} m_{l}\right)^{\frac{3}{2}}}{\left(2 \pi k_{B} T\right)^{3}\left(m_{k}+m_{l}\right)^{2}} \int_{\mathbb{R}^{3}} \exp \left(-\frac{M X_{*}^{2}}{2 k_{B} T}\right) d \mathbf{X}_{*} \\
\times \int_{\mathbb{R}^{3}}\left(\mathbf{a}_{k} \cdot \mathbf{V}_{*}\right) \mathbf{V}_{*} \exp \left(-\frac{\mu_{k l} V_{*}^{2}}{2 k_{B} T}\right) V_{*} \Theta\left(V_{*}-\frac{\Xi_{k l}}{c_{0}}\right) d \mathbf{V}_{*} .
\end{array}
$$

Setting $\left(\mathbf{a}_{k} \cdot \mathbf{X}_{*}\right) \mathbf{X}_{*}=a_{k} \cos \theta X_{*}^{2} \overrightarrow{\mathbf{x}}$, where $\overrightarrow{\mathbf{x}}$ is a unit vector, the first integral of the first term on the right hand side of 98 can be written in spherical coordinates as given below

$$
\begin{aligned}
\int_{\mathbb{R}^{3}} a_{k} \cos \theta X_{*}^{2} \overrightarrow{\mathbf{x}} \exp \left(-\frac{M X_{*}^{2}}{2 k_{B} T}\right) d \mathbf{X}_{*} \\
=a_{k} \int_{0}^{\infty} X_{*}^{4} \exp \left(-\frac{M X_{*}^{2}}{2 k_{B} T}\right) d X_{*}(\hat{\mathbf{x}} \int_{0}^{\pi} \sin ^{2} \theta \cos \theta d \theta \underbrace{\int_{0}^{2 \pi} \cos \phi d \phi}_{0} \\
+\hat{\mathbf{y}} \int_{0}^{\pi} \sin ^{2} \theta \cos \theta d \theta \underbrace{\int_{0}^{2 \pi} \sin \phi d \phi}_{0}+\hat{\mathbf{z}} \underbrace{\int_{0}^{\pi} \sin \theta \cos ^{2} \theta d \theta}_{\frac{2}{3}} \underbrace{\int_{0}^{2 \pi} d \phi}_{2 \pi})
\end{aligned}
$$

where $\hat{\mathbf{x}}, \hat{\mathbf{y}}, \hat{\mathbf{z}}$ are the Cartesian unit vectors in $\mathbb{R}^{3}$. Performing the integration with respect to $X_{*}$, we obtain

$$
\int_{\mathbb{R}^{3}}\left(\mathbf{a}_{k} \cdot \mathbf{X}_{*}\right) \mathbf{X}_{*} \exp \left(-\frac{M X_{*}^{2}}{2 k_{B} T}\right) d \mathbf{X}_{*}=\frac{\pi^{\frac{3}{2}}}{2}\left(\frac{2 k_{B} T}{M}\right)^{\frac{5}{2}} \mathbf{a}_{k} .
$$

The second integral of the first term on the right hand side of $(98)$ is similar to the first integral on the left hand side of 70 and is equal to $2 \pi\left(\frac{2 k_{B} T}{\mu_{k l}}\right)^{2} \Gamma\left(2, z_{i_{*}}\right)$, where $z_{i_{*}}=\left(\frac{\Xi_{k l}}{c_{0}}\right)^{2}\left\{\frac{\mu_{k l}}{2 k_{B} T}\right\}$.

The integral in $\mathbf{X}_{*}$ in the second and third terms on the right hand side of (98) vanishes. The first integral in the fourth term on the right hand side of 98 is similar to 69 and the second integral is similar to 93 and is equal to $\frac{2 \pi}{3}\left(\frac{2 k_{B} T}{\mu_{k l}}\right)^{3} \Gamma\left(3, z_{i_{*}}\right) \mathbf{a}_{k}$, where $z_{i_{*}}$ is as previously defined.

Putting together the previous computations and doing a little algebra, we obtain

$$
\mathcal{S}_{1}^{1}=\sigma_{i j}^{2} \alpha^{2} c_{k}^{\alpha} c_{l}^{\alpha} \frac{2}{M}\left(\frac{2 \pi k_{B} T}{\mu_{k l}}\right)^{\frac{1}{2}}\left[m_{k} \Gamma\left(2, z_{i_{*}}\right)+\frac{2}{3} m_{l} \Gamma\left(3, z_{i_{*}}\right)\right] \mathbf{u}_{k}^{\alpha} .
$$

Employing a similar strategy in the computation of $\mathcal{S}_{1}^{2}$, we obtain that

$$
\mathcal{S}_{1}^{2}=\sigma_{i j}^{2} \alpha^{2} c_{k}^{\alpha} c_{l}^{\alpha} \frac{1}{M}\left(\frac{\Xi_{k l}}{c_{0}}\right)^{2}\left(\frac{2 \pi \mu_{k l}}{k_{B} T}\right)^{\frac{1}{2}}\left[m_{k} \Gamma\left(1, z_{i_{*}}\right)+\frac{2}{3} m_{l} \Gamma\left(2, z_{i_{*}}\right)\right] \mathbf{u}_{k}^{\alpha} .
$$

Since $\mathcal{S}_{1}$ is the difference between $\mathcal{S}_{1}^{1}$ and $\mathcal{S}_{1}^{2}$, we obtain 


$$
\begin{aligned}
\mathcal{S}_{1}=\sigma_{i j}^{2} \alpha^{2} c_{k}^{\alpha} c_{l}^{\alpha} \frac{2}{M}\left(\frac{2 \pi k_{B} T}{\mu_{k l}}\right)^{\frac{1}{2}}[ & \left.m_{k} \Gamma\left(2, z_{i_{*}}\right)+\frac{2}{3} m_{l} \Gamma\left(3, z_{i_{*}}\right)\right] \mathbf{u}_{k}^{\alpha} \\
& \quad-\sigma_{i j}^{2} \alpha^{2} c_{k}^{\alpha} c_{l}^{\alpha} \frac{1}{M}\left(\frac{\Xi_{k l}}{c_{0}}\right)^{2}\left(\frac{2 \pi \mu_{k l}}{k_{B} T}\right)^{\frac{1}{2}}\left[m_{k} \Gamma\left(1, z_{i_{*}}\right)+\frac{2}{3} m_{l} \Gamma\left(2, z_{i_{*}}\right)\right] \mathbf{u}_{k}^{\alpha} .
\end{aligned}
$$

Similarly,

$$
\begin{aligned}
\mathcal{S}_{2}=2 \sigma_{i j}^{2} \alpha^{2} c_{k}^{\alpha} c_{l}^{\alpha} \frac{m_{l}}{M}\left(\frac{2 \pi k_{B} T}{\mu_{k l}}\right)^{\frac{1}{2}}\left[\Gamma\left(2, z_{i_{*}}\right)\right. & \left.-\frac{2}{3} \Gamma\left(3, z_{i_{*}}\right)\right] \mathbf{u}_{l}^{\alpha} \\
& \quad-\sigma_{i j}^{2} \alpha^{2} c_{k}^{\alpha} c_{l}^{\alpha} \frac{m_{l}}{M}\left(\frac{\Xi_{k l}}{c_{0}}\right)^{2}\left(\frac{2 \pi \mu_{k l}}{k_{B} T}\right)^{\frac{1}{2}}\left[\Gamma\left(1, z_{i_{*}}\right)-\frac{2}{3} \Gamma\left(2, z_{i_{*}}\right)\right] \mathbf{u}_{l}^{\alpha} .
\end{aligned}
$$

Since $\mathcal{S}$ is the sum of $\mathcal{S}_{1}$ and $\mathcal{S}_{2}$, we obtain

$$
\begin{aligned}
\mathcal{S} & =\sigma_{i j}^{2} \alpha^{2} c_{k}^{\alpha} c_{l}^{\alpha} \frac{2}{M}\left(\frac{2 \pi k_{B} T}{\mu_{k l}}\right)^{\frac{1}{2}}\left[m_{k} \Gamma\left(2, z_{i_{*}}\right)+\frac{2}{3} m_{l} \Gamma\left(3, z_{i_{*}}\right)\right] \mathbf{u}_{k}^{\alpha} \\
& -\sigma_{i j}^{2} \alpha^{2} c_{k}^{\alpha} c_{l}^{\alpha} \frac{1}{M}\left(\frac{\Xi_{k l}}{c_{0}}\right)^{2}\left(\frac{2 \pi \mu_{k l}}{k_{B} T}\right)^{\frac{1}{2}}\left[m_{k} \Gamma\left(1, z_{i_{*}}\right)+\frac{2}{3} m_{l} \Gamma\left(2, z_{i_{*}}\right)\right] \mathbf{u}_{k}^{\alpha} \\
& +2 \sigma_{i j}^{2} \alpha^{2} c_{k}^{\alpha} c_{l}^{\alpha} \frac{m_{l}}{M}\left(\frac{2 \pi k_{B} T}{\mu_{k l}}\right)^{\frac{1}{2}}\left[\Gamma\left(2, z_{i_{*}}\right)-\frac{2}{3} \Gamma\left(3, z_{i_{*}}\right)\right] \mathbf{u}_{l}^{\alpha} \\
& -\sigma_{i j}^{2} \alpha^{2} c_{k}^{\alpha} c_{l}^{\alpha} \frac{m_{l}}{M}\left(\frac{\Xi_{k l}}{c_{0}}\right)^{2}\left(\frac{2 \pi \mu_{k l}}{k_{B} T}\right)^{\frac{1}{2}}\left[\Gamma\left(1, z_{i_{*}}\right)-\frac{2}{3} \Gamma\left(2, z_{i_{*}}\right)\right] \mathbf{u}_{l}^{\alpha} .
\end{aligned}
$$

Now, we treat the last term on the right hand side of $(96)$. Observe that it can be written as the sum of the following two terms

$$
\begin{aligned}
& \mathcal{T}_{1}=-\alpha \sigma_{i j}^{2} \int_{\mathbb{R}^{3}} \int_{\mathbb{R}^{3}} \int_{\mathbb{S}_{+}^{2}} \mathbf{v}_{i}\left(\mathbf{a}_{i} \cdot \mathbf{v}_{i}\right) M_{i}^{\alpha} M_{j}^{\alpha} \Theta\left(\left\langle\epsilon, c_{0} \mathbf{v}_{i}-c_{0} \mathbf{v}_{j}\right\rangle-\Xi_{i j}\right)\left\langle\epsilon, \mathbf{v}_{i}-\mathbf{v}_{j}\right\rangle d \epsilon d \mathbf{v}_{j} d \mathbf{v}_{i}, \\
& \mathcal{T}_{2}=-\alpha \sigma_{i j}^{2} \int_{\mathbb{R}^{3}} \int_{\mathbb{R}^{3}} \int_{\mathbb{S}_{+}^{2}} \mathbf{v}_{i}\left(\mathbf{a}_{j} \cdot \mathbf{v}_{j}\right) M_{i}^{\alpha} M_{j}^{\alpha} \Theta\left(\left\langle\epsilon, c_{0} \mathbf{v}_{i}-c_{0} \mathbf{v}_{j}\right\rangle-\Xi_{i j}\right)\left\langle\epsilon, \mathbf{v}_{i}-\mathbf{v}_{j}\right\rangle d \epsilon d \mathbf{v}_{j} d \mathbf{v}_{i} .
\end{aligned}
$$

Equations (102) are similar to (97), so we can do exactly the same computations as we did above for $\mathcal{S}$, and we get at the end that

$$
\begin{aligned}
\mathcal{T} & =-\alpha^{2} \sigma_{i j}^{2} c_{i}^{\alpha} c_{j}^{\alpha} \frac{2}{M}\left(\frac{2 \pi k_{B} T}{\mu_{i j}}\right)^{\frac{1}{2}}\left[m_{i} \Gamma\left(2, z_{i}^{*}\right)+\frac{2}{3} m_{j} \Gamma\left(3, z_{i}^{*}\right)\right] \mathbf{u}_{i}^{\alpha} \\
& +\sigma_{i j}^{2} \alpha^{2} c_{i}^{\alpha} c_{j}^{\alpha} \frac{1}{M}\left(\frac{\Xi_{i j}}{c_{0}}\right)^{2}\left(\frac{2 \pi \mu_{i j}}{k_{B} T}\right)^{\frac{1}{2}}\left[m_{i} \Gamma\left(1, z_{i}^{*}\right)+\frac{2}{3} m_{j} \Gamma\left(2, z_{i}^{*}\right)\right] \mathbf{u}_{i}^{\alpha} \\
& -2 \alpha^{2} \sigma_{i j}^{2} c_{i}^{\alpha} c_{j}^{\alpha} \frac{m_{j}}{M}\left(\frac{2 \pi k_{B} T}{\mu_{i j}}\right)^{\frac{1}{2}}\left[\Gamma\left(2, z_{i}^{*}\right)-\frac{2}{3} \Gamma\left(3, z_{i}^{*}\right)\right] \mathbf{u}_{j}^{\alpha} \\
& +\sigma_{i j}^{2} \alpha^{2} c_{i}^{\alpha} c_{j}^{\alpha} \frac{m_{j}}{M}\left(\frac{\Xi_{i j}}{c_{0}}\right)^{2}\left(\frac{2 \pi \mu_{i j}}{k_{B} T}\right)^{\frac{1}{2}}\left[\Gamma\left(1, z_{i}^{*}\right)-\frac{2}{3} \Gamma\left(2, z_{i}^{*}\right)\right] \mathbf{u}_{j}^{\alpha}
\end{aligned}
$$

Finally, since $\mathcal{R}=0$, we obtain

$$
\mathcal{Q}_{i}=\mathcal{S}+\mathcal{T}
$$

with $\mathcal{S}$ and $\mathcal{T}$ given by 101 and 103 , respectively. 